\title{
A solar-like magnetic cycle on the mature K-dwarf 61 Cygni A (HD 201091)
}

\author{
S. Boro Saikia ${ }^{1}$, S. V. Jeffers ${ }^{1}$, J. Morin ${ }^{2}$, P. Petit ${ }^{3,4}$, C. P. Folsom ${ }^{5}$, S. C. Marsden ${ }^{6}$, J.-F. Donati ${ }^{3,4}$, R. Cameron ${ }^{7}$, \\ J. C. Hall ${ }^{8}$, V. Perdelwitz ${ }^{9}$, A. Reiners ${ }^{1}$, and A. A. Vidotto ${ }^{10,11}$
}

1 Institut für Astrophysik, Universität Göttingen, Friedrich Hund Platz 1, 37077 Göttingen, Germany e-mail: sudeshna@astro.physik.uni-goettingen.de

2 LUPM-UMR 5299, CNRS \& Université Montpellier, place Eugène Bataillon, 34095 Montpellier Cedex 05, France

3 CNRS, Institut de Recherche en Astrophysique et Planétologie, 14 avenue Edouard Belin, 31400 Toulouse, France

${ }^{4}$ Université de Toulouse, UPS-OMP, Institut de Recherche en Astrophysique et Planétologie, 31400 Toulouse, France

5 IPAG, UJF-Grenoble 1/CNRS-INSU, UMR 5274, 38041 Grenoble, France

${ }^{6}$ Computational Engineering and Science Research Centre, University of Southern Queensland, 4350 Toowoomba, Australia

7 Max-Planck-Institut für Sonnensystemforschung, Justus-von-Liebig-Weg 3, 37077 Göttingen, Germany

${ }^{8}$ Lowell Observatory, 1400 West Mars Hill Road, Flagstaff, AZ 86001, USA

9 Hamburger Sternwarte, Gojenbergsweg 112, 21029 Hamburg, Germany

10 Observatoire de Genève, Université de Genève, Chemin des Maillettes 51, 1290 Sauverny, Switzerland

11 School of Physics, Trinity College Dublin, The University of Dublin, Dublin-2, Ireland

Received 5 February 2016 / Accepted 22 June 2016

\section{ABSTRACT}

\begin{abstract}
Context. The long-term monitoring of magnetic cycles in cool stars is a key diagnostic in understanding how dynamo generation and amplification of magnetic fields occur in stars similar in structure to the Sun.

Aims. We investigated the temporal evolution of a possible magnetic cycle of $61 \mathrm{Cyg} \mathrm{A}$. The magnetic cycle is determined from $61 \mathrm{Cyg}$ A's large-scale field over its activity cycle using spectropolarimetric observations and compared to the solar large-scale magnetic field. Methods. We used the tomographic technique of Zeeman Doppler imaging (ZDI) to reconstruct the large-scale magnetic geometry of 61 Cyg A over multiple observational epochs spread over a time span of nine years. We investigated the time evolution of the different components of the large-scale field and compared it with the evolution of the star's chromospheric activity by measuring the flux in three different chromospheric indicators: $\mathrm{Ca}$ II $\mathrm{H} \& \mathrm{~K}, \mathrm{H} \alpha$ and $\mathrm{Ca}$ II infrared triplet lines. We also compared our results with the star's coronal activity using XMM-Newton observations.

Results. The large-scale magnetic geometry of $61 \mathrm{Cyg}$ A exhibits polarity reversals in both poloidal and toroidal field components, in phase with its chromospheric activity cycle. We also detect weak solar-like differential rotation with a shear level similar to that of the Sun. During our observational time span of nine years, $61 \mathrm{Cyg}$ A exhibits solar- like variations in its large-scale field geometry as it evolves from minimum activity to maximum activity and vice versa. During its activity minimum in epoch 2007.59, ZDI reconstructs a simple dipolar geometry which becomes more complex when it approaches activity maximum in epoch 2010.55. The radial field flips polarity and reverts back to a simple geometry in epoch 2013.61. The field is strongly dipolar and the evolution of the dipole component of the field is reminiscent of solar behaviour. The polarity reversal of the large-scale field indicates a magnetic cycle that is in phase with the chromospheric and coronal cycle.
\end{abstract}

Key words. dynamo - stars: activity - stars: chromospheres - stars: magnetic field - stars: solar-type

\section{Introduction}

High resolution observations of the Sun, including over $30 \mathrm{yr}$ of synoptic magnetic field maps, have revealed a coherent picture of the way in which the large-scale magnetic field evolves over the course of the 22-yr solar magnetic cycle. At minimum, the field is concentrated at the polar regions and is mostly axisymmetric with respect to the rotation axis. At activity maximum, the field is no longer concentrated at the polar regions and is restricted to lower latitudes (Hathaway 2010). When the next minimum occurs, the polar field reappears but with the opposite sign to that observed in the previous minimum at the start of the cycle. This cyclic process repeats every $11 \mathrm{yr}$ and it takes $22 \mathrm{yr}$ for the polar magnetic fields to revert back to the same polarity, forming a 22yr magnetic cycle. During the course of the solar magnetic cycle the geometry of the large-scale field of the Sun also changes dramatically. The Sun varies from an almost dipolar geometry during cycle minimum to a more complex geometry during cycle maximum (Sanderson et al. 2003; DeRosa et al. 2012). A recent study by DeRosa et al. (2012) has revealed that during cycle minimum the dipolar component of the large-scale solar field dominates the other components. During polarity reversals the quadrupolar and octopolar component of the large-scale field dominates the dipolar component.

When it comes to other solar-type stars, observations do not have even a fraction of the spatial and temporal resolution of the solar observations. Magnetic cycles in other cool stars have been investigated by using two well known proxies of magnetic activity: chromospheric activity (Baliunas et al. 1995; Hall 2008) and coronal activity (Pevtsov et al. 2003; Güdel 2004). The first long-term monitoring of chromospheric activity in solar-type dwarfs was carried out at the Mount Wilson observatory (Wilson 1978; Duncan et al. 1991; Baliunas et al. 1995), where emission in the line cores of $\mathrm{Ca}$ II $\mathrm{H} \& \mathrm{~K}$ lines was measured. These observations revealed that different solar-type 
stars tend to exhibit different levels of activity variation: irregular activity variations in fast rotating young stars, cyclic activity in comparatively older slowly rotating solar-type stars, and a possible Maunder-minimum in some stars (Baliunas et al. 1995). Our investigation of chromospheric activity cycles of Mount Wilson and HARPS stars has also confirmed that solarlike cycles are more likely for slow rotating stars than for fast rotating stars (Boro Saikia et al., in prep.). A further analysis of the Maunder-minimum candidates from the Mt Wilson survey by Schröder et al. (2013) reveals them to be slightly more evolved than the Sun. Coronal activity of cool stars, on the other hand is not intensively monitored compared to their chromospheric activity. Analysis by Hempelmann et al. (1996) produced weak statistical evidence of coronal cycles in cool stars, and since then coronal activity cycles have been detected for a few selected G and K dwarfs. For example, Favata et al. (2008) reported the presence of a coronal activity cycle for the binary system HD81809. Cyclic X-ray activity was also detected for our target star 61 Cyg A (Robrade et al. 2012) and the K1 dwarf $\alpha$ Cen B (Robrade et al. 2012; Ayres 2015). The shortest coronal activity cycle of $1.6 \mathrm{yr}$ was reported for the young active Sun $\iota$ Horologii (Sanz-Forcada et al. 2013), where the coronal activity cycle is in phase with its short chromospheric activity cycle.

Although these indirect proxies are reliable indicators of magnetic activity in cool stars, they do not provide any direct information on the strength or orientation of the large-scale magnetic field. Direct measurements of the surface magnetic field rely on the Zeeman effect. By measuring the broadening of spectral lines caused by the Zeeman effect acting on unpolarised spectra (Robinson 1980; Saar 1996; Reiners \& Basri 2006), the integrated unsigned magnetic flux, averaged over the entire disk of the star, can be measured. One advantage of this technique is that the small-scale magnetic features also contribute to the total field measurements. This technique does not provide information on the large-scale magnetic field geometry. However the tomographic technique Zeeman Doppler imaging (ZDI; Semel 1989; Donati et al. 1997) uses spectropolarimetric observations to reconstruct the stellar surface magnetic field geometry. Due to cancellation effects this technique is insensitive to small-scale multipolar features, as the overall polarisation signatures of the multipolar field are cancelled out and only the large-scale field is reconstructed. As the ZDI technique provides information about the vector magnetic field, it can provide invaluable insights into the large-scale field geometry as well as the temporal evolution and polarity reversals of the large-scale field.

The large-scale magnetic field of several solar-type stars reconstructed using ZDI over multiple epochs, has revealed their strongly varying magnetic geometry. For the G7 dwarf $\xi$ Bootis A $\left(0.86 M_{\odot}, T_{\text {eff }}=5551 \mathrm{~K}\right.$; Morgenthaler et al. 2012), the K2 dwarf $\epsilon$ Eridani $\left(0.856 M_{\odot}, T_{\text {eff }}=5146 \mathrm{~K}\right.$; Jeffers et al. 2014), and the G0 dwarf HN Peg $\left(1.085 M_{\odot}, T_{\text {eff }}=5974 \mathrm{~K}\right.$; Boro Saikia et al. 2015) rapidly varying field geometry was detected, with surprising appearances and disappearances of the azimuthal field for HN Peg and $\epsilon$ Eridani. A two-year magnetic cycle was revealed for the planet hosting F7 dwarf $\tau$ Bootis (1.42 $M_{\odot}, T_{\text {eff }}=6360 \mathrm{~K}$; Donati et al. 2008; Fares et al. 2009). $\tau$ Boo is a hot Jupiter host where the planet is orbiting at 0.049 AU. No correlation was detected between the large-scale polarity switch and its chromospheric activity (Fares et al. 2009). Further monitoring of $\tau$ Boo over three epochs by Fares et al. (2013) has confirmed the previously determined two-year magnetic cycle. Recent new analysis of $\tau$ Boo polarised spectra (Mengel et al. 2016) shows a 3:1 ratio between the magnetic and chromospheric cycles. A magnetic cycle was also detected for the G0
Table 1. Summary of the physical parameters of $61 \mathrm{Cyg}$ A.

\begin{tabular}{llc}
\hline \hline Parameters & HD 201091 & References \\
\hline Effective temperature, $T_{\text {eff }}(\mathrm{K})$ & $4545 \pm 40$ & this work \\
Spectral type & K5V & 1 \\
$\log g$ & $4.75 \pm 0.10$ & this work \\
Radius, $R_{\odot}$ & $0.665 \pm 0.005$ & 1 \\
Mass, $M_{\odot}$ & 0.66 & 1 \\
Rotation period, days & $35.7 \pm 1.9$ & this work \\
.. & $34.2 \pm 3.7$ & this work \\
Inclination & $70^{\circ}$ & this work \\
$B-V$ & 1.069 & 2 \\
Age, Gyr & 6.0 & 3 \\
.. & 3.6 & 4 \\
.. & 2.0 & 5 \\
.. & 1.331 & 6 \\
\hline
\end{tabular}

References. (1) Kervella et al. (2008); (2) Perryman et al. (1997); (3) Kervella et al. (2008); (4) Mamajek \& Hillenbrand (2008); (5) Barnes (2007); (6) Marsden et al. (2014). The rotation period of $35.7 \pm 1.9$ is derived from chromospheric activity measurements and the period of $34.2 \pm 3.7$ is derived from ZDI (see text for more details).

dwarf HD $78366\left(1.34 M_{\odot}, T_{\text {eff }}=6014 \mathrm{~K}\right.$; Morgenthaler et al. 2011), where the radial component of the magnetic field exhibits polarity reversals indicating a possible three-year cycle. Polarity reversals were observed in the large-scale field of the G2 dwarf HD $190771\left(0.96 M_{\odot}, T_{\text {eff }}=5834 \mathrm{~K}\right.$; Petit et al. 2009; Morgenthaler et al. 2011). However, the variability of the large-scale field of HD 190771 is more complex as the polarity reversal in the azimuthal field reported by Petit et al. (2009) is not detected in subsequent epochs. Instead polarity reversal is reported in the radial component of the magnetic field in the subsequent epochs as shown by Morgenthaler et al. (2011). Polarity reversals of the large-scale field have been detected only for a select few cool stars, out of which none exhibit a magnetic cycle period equivalent to the star's chromospheric cycle period.

This paper investigates the variability of the large-scale magnetic field geometry of the solar-type K5 dwarf 61 Cyg A, using a time series of spectropolarimetric observations over nine years. We also investigate the correlation between its mean magnetic field, chromospheric activity, and coronal activity. The stellar parameters of $61 \mathrm{Cyg} \mathrm{A}$ are discussed in Sect. 2, followed by instrumental setup and data reduction in Sect. 3. Section 4 covers both direct and indirect field detection. The large-scale magnetic geometry is discussed in Sect. 5, followed by the long-term evolution of the magnetic and activity cycle in Sect. 6 . The results are discussed in Sect. 7 and finally the summary in Sect. 8.

\section{Physical properties of 61 Cyg A}

A solar-type dwarf of spectral type K5, 61 Cyg A forms the well known visual binary $61 \mathrm{Cyg}$ together with the K7 dwarf $61 \mathrm{Cyg}$ B. The binary $61 \mathrm{Cyg}$ is a northern system with a semimajor axis of approximately $24^{\prime \prime}$ (Malkov et al. 2012). It is the first stellar object to have its parallax measured (Bessel 1838). $61 \mathrm{Cyg}$ A has a parallax of $287.13 \pm 1.51$ mas (Perryman et al. 1997) and is at a distance of approximately 3.5 pc. It is a slow rotator with a rotation period of $35.7 \pm 1.9$ days as shown in Table 1, where the rotation period is obtained from long-term chromospheric activity measurements. We also derive a rotation period of $34.2 \pm 3.7$ days using ZDI (see Sect. 5.3 for more details). $61 \mathrm{Cyg} \mathrm{A}$ has a radius of $0.665 \pm 0.005 R_{\odot}$ (Kervella et al. 2008), where the radius was determined using interferometric 
data, and is in agreement with the radius of $0.62 R_{\odot}$ (Takeda et al. 2007) calculated by using a stellar evolution code. Using the same code and stellar evolutionary tracks, Takeda et al. (2007) also determined a mass of $0.660 M_{\odot}$. The angular diameter of $1.775 \pm 0.013$ mas (Kervella et al. 2008) and the bolometric flux of $0.3844 \times 10^{-9}$ (Mann et al. 2013) can be used to determine a $T_{\text {eff }}$ of $4374 \mathrm{~K}$. Applying to our highest $\mathrm{S} / \mathrm{N}$ spectra our automatic spectral classification tool ${ }^{1}$, we find that the photosphere temperature, logarithmic gravity, and metallicity of $61 \mathrm{Cyg}$ A are respectively equal to $4545 \pm 40 \mathrm{~K}, 4.75 \pm 0.10$, and $-0.18 \pm 0.05$. The $T_{\text {eff }}$ from our analysis agrees with the literature value of $4525 \pm 140 \mathrm{~K}$ (Takeda et al. 2007) with improved accuracy. Using the radius and rotation period from Table 1, we determine an equatorial $v_{\mathrm{e}}$ of $0.94 \mathrm{kms}^{-1}$. This suggests that the $v \sin i$ of 61 Cyg A should be $\leq 0.94 \mathrm{kms}^{-1}$. A chromospheric age of 1.331 Gyr was determined by Marsden et al. (2014) from the activity age relationship of Wright et al. (2004). Using chromospheric activity and rotation relation, Mamajek \& Hillenbrand (2008) determine an age of 3.6 Gyr. In contrast, Kervella et al. (2008) predicted an age of approximately 6 Gyr using evolutionary models combined with interferometric radius measurements. The age estimates of $61 \mathrm{Cyg}$ A from gyrochronology puts its age at approximately 2 Gyr (Barnes 2007). The stellar parameters of 61 Cyg A are summarised in Table 1.

$61 \mathrm{Cyg} \mathrm{A}$ is a moderately active star and was included in the long-term Mount Wilson survey (Duncan et al. 1991). It was discovered to exhibit an activity cycle of $7.3 \pm 0.1 \mathrm{yr}$ (Baliunas et al. 1995). 61 Cyg A was also included in the Lowell Observatory long-term survey, where it was classified as a star with solar-type variability (Hall et al. 2007; Lockwood et al. 2007). A coronal activity cycle of approximately seven years has also been detected for 61 Cyg A (Robrade et al. 2012) using X-ray observations, where the coronal activity cycle is in phase with the chromospheric activity cycle.

\section{Instrumental setup and data reduction}

\subsection{Optical data}

The spectropolarimetric data was collected as part of the BCool collaboration $^{2}$ using the NARVAL spectropolarimeter, at the 2.0 $\mathrm{m}$ Telescope Bernard Lyot at Pic du Midi Observatory. NARVAL is equipped with a cross dispersed echelle spectrograph, which is a twin of the EsPaDOnS spectropolarimeter (Donati 2003) at the $3.6 \mathrm{~m}$ Canada-France-Hawaii telescope (CFHT). It covers the full optical wavelength range from $370 \mathrm{~nm}$ to $1000 \mathrm{~nm}$, with a resolving power of approximately 65000 (Aurière 2003). The spectropolarimetric observations of $61 \mathrm{Cyg}$ A were obtained by combining four successive sub-exposures, taken with different half wave rhomb angles. The data is reduced on-site by using a fully automated reduction package, LIBRE-ESPRIT, which generates both intensity (Stokes $I$ ) and circularly polarised (Stokes $V$ ) spectra. LIBRE-ESPIRIT is based on the reduction pipeline ESPIRIT developed by Donati et al. (1997). The reduction package also calculates a diagnostic Null profile, which is a control profile, by combining the four sub-exposures in such a way that any polarisation signal is cancelled out. The output from the reduction pipeline is continuum normalised. Table A.1 shows the journal of observations of $61 \mathrm{Cyg}$ A.

Seven data sets comprising seven epochs spanning nine years of both Stokes $I$ and $V$ spectra, were obtained covering epochs

\footnotetext{
1 Our spectral classification tool is inspired from that of Valenti \& Fischer (2005) and is discussed in a previous paper (Donati et al. 2012).

2 http://bcool.ast.obs-mip.fr/Bcool
}

2007.59, 2008.64, 2010.55, 2012.54, 2013.61, 2014.61, and 2015.54. Each epoch contains between nine and sixteen spectra. The spectropolarimetric data from the 2012.54 epoch was discarded due to polarisation anomalies in one of the polarisation rhombs at NARVAL during 2011-2012 ${ }^{3}$. The azimuthal angle of the rhomb was wrong during that epoch, leading to incorrect polarisation measurements.

For active cool stars, Zeeman-induced circular polarisation has too low an amplitude to be detected in individual spectral lines. Hence, the technique of least square deconvolution (LSD) is applied to the spectra of $61 \mathrm{Cyg}$ A. LSD is a multiline technique which assumes a similar line profile for all magnetically sensitive lines in a spectra and generates an averaged line profile by deconvolving the stellar spectra to a line mask (Donati et al. 1997; Kochukhov et al. 2010). We used a K5 mask consisting of approximately 12000 lines to compute the LSD Stokes $I$ and Stokes $V$ profiles of $61 \mathrm{Cyg}$ A. The LSD technique used in this paper is detailed in Marsden et al. (2014).

\subsection{X-ray data}

$61 \mathrm{Cyg} \mathrm{A}$ is a strong X-ray source and has been continuously monitored with XMM-Newton since 2002 with a cadence of two observations a year (see e.g. Hempelmann et al. 2006; and Robrade et al. 2012). Therefore, in order to check for periodicity in X-ray luminosity we obtained all public data sets from the XMM-Newton Science Archive. Data reduction was performed with the XMM-Newton Science Analysis System version 10.0, and XSPEC 12.7.1. In order to avoid contamination from 61 Cyg B, source photons were extracted with a $15^{\prime \prime}$ aperture, while background levels were estimated with a $45^{\prime \prime}$ aperture in source-free regions. Time intervals during which obvious flaring occurred were omitted. The spectra of all three EPIC detectors (MOS $1 \& 2$ and PN) were then fitted simultaneously in the $0.2-2.0 \mathrm{keV}$ range with a three-temperature APEC model.

\section{Magnetic field detection: direct and indirect approach}

\subsection{Mean longitudinal magnetic field}

We measure the line-of-sight component of the magnetic field, averaged over the stellar surface for each observation using Stokes $V$ and Stokes $I$ LSD profile. The mean longitudinal field is measured from the Stokes $V$ and $I$ profile (Donati et al. 1997) as shown in Eq. (1),

$B_{l}(\mathrm{G})=-2.14 \times 10^{11} \frac{\int v V(v) \mathrm{d} v}{\lambda_{0} g c \int\left(I_{\mathrm{c}}-I(v)\right) \mathrm{d} v}$

where $B_{l}$ represents the mean longitudinal magnetic field of 61 Cyg A in Gauss, $\lambda_{0}=630 \mathrm{~nm}$ is the central wavelength of the LSD profile, $g=1.22$ is the Lande factor of the line list, $c$ is the speed of light in $\mathrm{km} \mathrm{s}^{-1}, v$ is the radial velocity in $\mathrm{km} \mathrm{s}^{-1}$, and $I_{\mathrm{c}}$ is the normalised continuum intensity. A velocity range of $\pm 20 \mathrm{kms}^{-1}$ from the line centre was used. The velocity window was selected so that the entire signal could be included. If a smaller integration window is used, then the wings of the signal are ignored which may result in an underestimated value of $B_{l}$. We also calculated the magnetic field from the Null profile $\left(N_{l}\right)$. The error estimates were made by propagating the uncertainties calculated by the reduction pipeline, as mentioned in

\footnotetext{
http://spiptbl.bagn.obs-mip.fr/Actualites/ Anomalies-de-mesures
} 
Marsden et al. (2014). Table A.2 shows the values of $B_{l}, N_{l}$, and the associated uncertainties for each observation.

\subsection{Chromospheric activity as a proxy of magnetic activity}

61 Cyg A is known to exhibit cyclic chromospheric activity of approximately seven years (Baliunas et al. 1995). In order to investigate the chromospheric activity of $61 \mathrm{Cyg} \mathrm{A}$, we monitored the fluxes in three chromospheric indicators: Ca II $\mathrm{H} \& \mathrm{~K}, \mathrm{H} \alpha$, and the Ca II infrared triplet. These three chromospheric indicators are well known proxies of stellar activity, where the different indicators have different sensitivities to the various chromospheric features such as plages, networks, flares, and filaments (Meunier \& Delfosse 2009). The Ca II H\&K lines are resonance lines which are dependent on the local chromospheric temperature and electron density (Jefferies \& Thomas 1959). Contrary to the $\mathrm{Ca}$ II H\&K lines, the $\mathrm{H} \alpha$ line is a Balmer line which is insensitive to the local chromospheric conditions but more sensitive to the radiation temperature (Jefferies \& Thomas 1959). Hence, it is important to explore the different chromospheric tracers to obtain as complete a picture as possible of the chromosphere of an active cool star.

\subsubsection{S-index}

The S-index has been one of the most widely used proxies of magnetic activity since its introduction in the long-term Mount Wilson survey (Wilson 1978; Duncan et al. 1991; Baliunas et al. 1995). We measure the S-index of 61 Cyg A by using two triangular band passes with a FWHM of $0.1 \mathrm{~nm}$, centred at the line cores of the Ca II K and $\mathrm{H}$ line at $393.3663 \mathrm{~nm}$ and $396.8469 \mathrm{~nm}$ respectively. The fluxes in the nearby continuum are measured by using two $2 \mathrm{~nm}$ wide rectangular band passes at the blue and red side of the $\mathrm{K}$ and $\mathrm{H}$ lines at $390.107 \mathrm{~nm}$ and $400.107 \mathrm{~nm}$ respectively (Duncan et al. 1991; Wright et al. 2004). In order to calibrate our measurements to the original Mount Wilson scale, we use the calibration coefficients calculated by Marsden et al. (2014), which is used in the S-index formula as shown in Eq. (2),

S-index $=\frac{\alpha F_{H}+\beta F_{K}}{\gamma F_{R}+\delta F_{V}}+\phi$

where $F_{H}, F_{K}, F_{R}$, and $F_{V}$ are the flux in the $H, K, R$, and $V$ bands and $\alpha=12.873, \beta=2.502, \gamma=8.877, \delta=4.271$, and $\phi=1.183 \mathrm{e}-03$ are the calibration coefficients respectively. The uncertainties associated with the S-index measurements are obtained by carrying out error propagation and are tabulated in Table A.2.

Figure 1 shows the rotational variability of both the $B_{l}$ and the $\mathrm{S}$-index as a function of the rotational phase for each epoch, allowing us to study variability on the rotation timescale. Overall, 61 Cyg A exhibits a stronger rotational variability in its longitudinal magnetic field during epochs 2007.59, 2010.55 and 2015.54 compared to epochs 2008.64, 2013.61 and 2014.61. During our observational time span the longitudinal field $B_{l}$ shows a change in sign which indicates that the field is nonaxisymmetric, except in epochs 2013.61 and 2014.61 which show signs of a more axisymmetric field. The $\mathrm{S}$-index exhibits low rotational dependence, indicating a simple magnetic geometry. However, one should be careful in interpreting the results because the rotational variations might be hidden due to poor phase coverage in certain epochs such as 2012.54. The longitudinal field could not be measured for epoch 2012.54, due to the unavailability of Stokes $V$ observations as already mentioned in the previous section.

61 Cyg A was part of the original Mount Wilson survey and was observed for three decades at the Mount Wilson observatory from 1961 to 1991 (Baliunas et al. 1995). A continuation of the Mount Wilson survey is being currently carried out at the Lowell observatory (Hall et al. 2007) and all available S-index data of 61 Cyg A together with our measurements are shown in Fig. 2. It shows the temporal variation of the S-index of 61 Cyg A calibrated to the Mount Wilson scale over a time span of $48 \mathrm{yr}$. At the beginning of our observational data in epoch 2007.59, $61 \mathrm{Cyg}$ A exhibits low activity and it increases to its maximum during epoch 2010.55. The chromospheric activity is low in epoch 2013.61, which indicates it is close to activity minimum. The activity in epoch 2014.61 is also very low but it increases again during epoch 2015.54. An activity cycle period of 7.2 $\pm 1.3 \mathrm{yr}$ was measured by taking a "generalised" Lomb-Scargle periodogram (Lomb 1976; Scargle 1982; Zechmeister \& Kürster 2009) on all available $S$-index data. This cycle period agrees with the activity cycle period of $7.3 \pm 0.1 \mathrm{yr}$, measured using only the Mount Wilson data (Baliunas et al. 1995).

\subsection{2. $\mathrm{H} \alpha$-index}

We measured the variability of the chromospheric activity of 61 Cyg A using $\mathrm{H} \alpha$ as an activity tracer for each epoch of this analysis. We employed the technique used by Gizis et al. (2002), where a rectangular band pass of $0.36 \mathrm{~nm}$ width was used at the $\mathrm{H} \alpha$ line at $656.285 \mathrm{~nm}$. Two rectangular band passes $H_{\text {blue }}$ and $H_{\text {red }}$ of $0.22 \mathrm{~nm}$ width were used to measure the nearby continuum,

$\mathrm{H} \alpha$-index $=\frac{H \alpha}{H_{\text {blue }}+H_{\text {red }}}$

where $H \alpha, H_{\text {blue }}$, and $H_{\text {red }}$ refers to different band passes used.

\subsubsection{CalRT-index}

The CaIRT-index of 61 Cyg A was measured by taking $0.2 \mathrm{~nm}$ wide rectangular band passes centred at the Ca II triplet lines at $849.8023 \mathrm{~nm}, 854.2091 \mathrm{~nm}$, and $866.241 \mathrm{~nm}$ respectively. The nearby continuum is measured by taking two $0.5 \mathrm{~nm}$ band passes $\mathrm{IR}_{\text {red }}$, and $\mathrm{IR}_{\text {blue }}$ at $870.49 \mathrm{~nm}$, and $847.58 \mathrm{~nm}$ respectively (Marsden et al. 2014), as shown in Eq. (4),

CaIRT-index $=\frac{\mathrm{IR} 1+\mathrm{IR} 2+\mathrm{IR} 3}{\mathrm{IR}_{\mathrm{red}}+\mathrm{IR}_{\text {blue }}}$

where IR1, IR2, and IR3 represent flux in the Ca II infra-red triplet lines. The continuum flux is represented by $\mathrm{IR}_{\text {red }}$ and $\mathrm{IR}_{\text {blue }}$ respectively.

The $\mathrm{H} \alpha$ and Ca IRT lines are in absorption throughout our observations. The $\mathrm{H} \alpha$-index variability as a function of the rotational phase is shown in Fig. 3. The rotational variability of the CaIRT-index is also shown in Fig. 3. Surprisingly the $\mathrm{H} \alpha$ and Ca II IRT indices do not show the strong variations shown by the $\mathrm{S}$-index. This might indicate that the magnetic field is not strong enough for these lines to go into emission explaining such low variations as compared to the $\mathrm{S}$-index. The uncertainties are obtained by carrying out error propagation and are shown in Table A.2. 

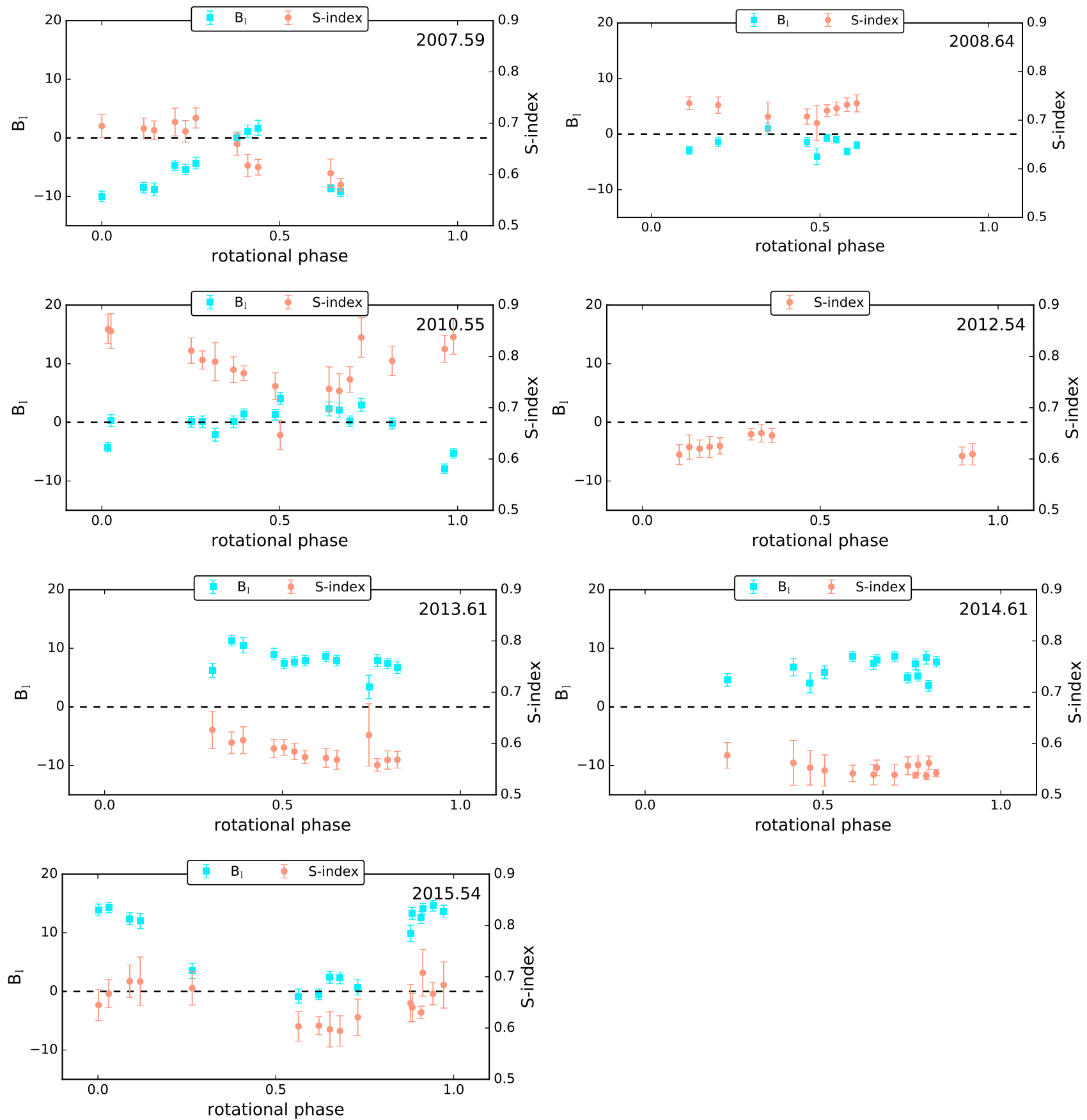

Fig. 1. Variability of the longitudinal field $\left(B_{l}\right)$ (left $y$-axis) and S-index (right $y$-axis) as a function of rotational phase for each epoch (2007.59, 2008.64, 2010.55, 2012.54, 2013.61, 2014.61 and 2015.54). For epoch 2012.54 only the S-index is plotted, as the Stokes $V$ observations were not available (see Sect. 3). The subplots are all on the same scale for easier comparison between epochs, and the dashed line represents $B_{l}=0$. The scale was chosen based on the minimum and maximum value.

\subsubsection{Coronal activity}

The X-ray luminosity variation of $61 \mathrm{Cyg} \mathrm{A}$ is shown in Fig. 5, where the data up to end of 2011 were previously published by Robrade et al. (2012) and the results from 2012 onward are derived for the first time here. While comparison to the results of Robrade et al. (2012) yields minor discrepancies caused by the different data fitting approaches, the overall picture is consistent. A clear cyclic variation in the X-ray luminosity is recovered with a cycle period of $6.6 \pm 0.5 \mathrm{yr}$. This is in good agreement with the chromospheric cycle of $7.2 \pm 1.3 \mathrm{yr}$.

\subsubsection{Correlation between activity indicators and longitudinal field}

As shown in Fig. 4, a possible weak anti-correlation between the absolute value of the longitudinal magnetic field $\left|B_{l}\right|$ and the $\mathrm{S}$-index is detected. The field measurements are the weakest in epoch 2010.55, when the chromospheric activity is at a maximum in our observations, and are the strongest close to minimum chromospheric activity. The average of the absolute longitudinal magnetic field $\left(\left|B_{l}\right|\right)$ per epoch is strongest in epoch 2015.54 with $9.3 \pm 5.9 \mathrm{G}$, and is weakest in epoch 2010.55 with $0.6 \pm 3.1 \mathrm{G}$. On the contrary strong correlation is detected between the three 


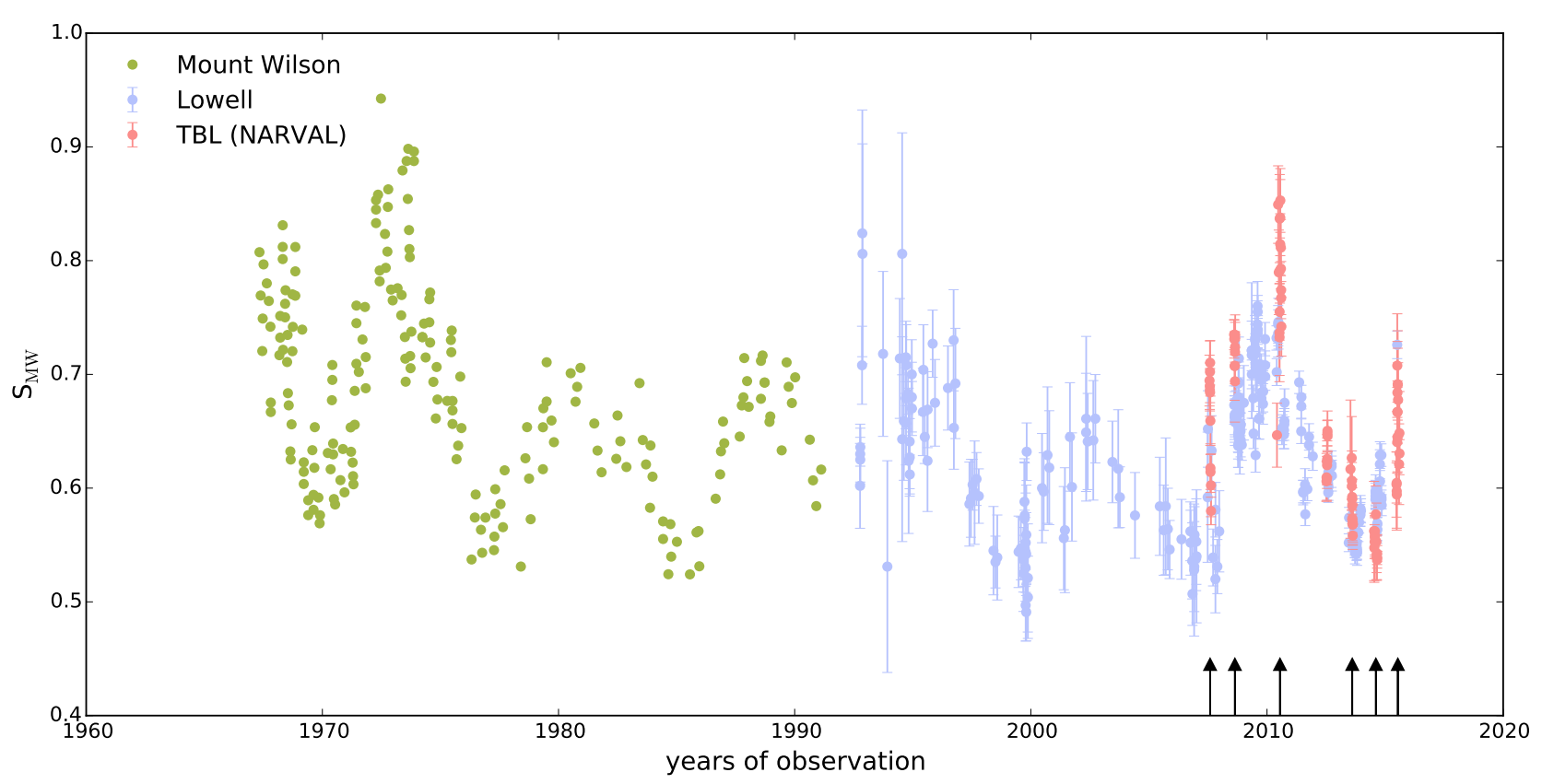

Fig. 2. Long-term chromospheric activity of $61 \mathrm{Cyg}$ A shown as S-index, which is calibrated to the Mount Wilson S-index. The arrow marks represent epochs for which the large-scale field map is reconstructed using ZDI.

chromospheric activity indicators as shown in Fig. 4. The CaIRTindex exhibits a slightly stronger correlation with the S-index $(r=0.7)$ compared to the $\mathrm{H} \alpha$-index $(r=0.6)$, which is not surprising as those two indicators are sensitive to similar chromospheric features. A strong correlation between the S-index and the CaIRT-index was also detected in the G dwarf $\xi$ Bootis A by Morgenthaler et al. (2012). Correlation between the $\log R_{\mathrm{HK}}^{\prime}$ and CaIRT-index was also studied for 170 solar-type stars as part of the BCool snapshot survey (Marsden et al. 2014). The $\log R_{\mathrm{HK}}^{\prime}$ is also an indicator of stellar activity where the photospheric contribution is corrected for the S-index (Wright et al. 2004).

\section{Large-scale magnetic field geometry}

The large-scale surface magnetic geometry of $61 \mathrm{Cyg} \mathrm{A}$ is reconstructed using the ZDI technique. ZDI is an inverse tomographic technique which reconstructs the stellar magnetic geometry from a time series of Stokes $V$ LSD profiles using maximum entropy as a regularisation technique (Skilling \& Bryan 1984). The ZDI version used in this paper reconstructs the magnetic geometry into its poloidal (potential field) and toroidal (nonpotential field) components expressed as spherical harmonic decomposition (see Donati et al. 2006).

If the total surface magnetic field is a combination of poloidal and toroidal field, it can be formalised into three field components $B_{r}, B_{\theta}$, and $B_{\phi} . B_{r}$ is the radial component of the field and is entirely poloidal, described by the spherical harmonics coefficient $\alpha_{l, m}$, where $l$ and $m$ are the order and degree respectively. $B_{\theta}$ is the meridional component of the total field and $B_{\phi}$ is the azimuthal component of the total field. $B_{\theta}$ and $B_{\phi}$ can be described by a combination of the spherical harmonics coefficients $\beta_{l, m}$ and $\gamma_{l, m}$ (Donati et al. 2006, see Sect.5.1). The order of the spherical harmonics coefficients determines if the field is simple dipolar or complex, and the degree determines if the field is axisymmetric or non-axisymmetric. For example, $l=1, m=0$ denotes the axisymmetric dipolar field. The quadrupolar and octopolar modes are denoted by higher order $l=2$ and $l=3$ respectively. The surface magnetic map and the associated Stokes $V$ spectra of a star can be derived using the spherical harmonics coefficients. ZDI tackles the inverse problem and reconstructs the large-scale surface magnetic field geometry by comparing model Stokes $V$ spectra to the observed Stokes $V$ spectra.

For 61 Cyg A we use a stellar model of 5000 grid points, where each grid point is associated with both a local Stokes $V$ and Stokes $I$ profile. A solar-type limb darkening law is used in the stellar model and synthetic Stokes $V$ profiles are computed for all rotational phases. Each of these local Stokes $V$ profiles are calculated under the weak field assumption and a uniform Stokes $I$ profile is assumed over the entire photosphere. Using maximum entropy as a regularisation scheme (Skilling \& Bryan 1984), ZDI reconstructs $\alpha_{l, m}, \beta_{l, m}$, and $\gamma_{l, m}$ by iteratively comparing the synthetic Stokes $V$ profiles to the observed Stokes $V$ profiles.

\subsection{Vector magnetic field}

61 Cyg A exhibits chromospheric variability on rotational timescales, indicating a high stellar inclination. We choose an inclination angle of $70^{\circ}$, which together with a rotation period of 34.2 days and a stellar radius of $0.665 R_{\odot}$, results in a $v \sin i$ of $0.92 \mathrm{~km} \mathrm{~s}^{-1}$. This $v \sin i$ is consistent with the $v_{\mathrm{e}}$ of $0.94 \mathrm{~km} \mathrm{~s}^{-1}$ mentioned in Sect. 2. We therefore used the $v \sin i$ of $0.92 \mathrm{~km} \mathrm{~s}^{-1}$ and an inclination angle of $70^{\circ}$ in our magnetic field reconstructions. We tested a range of spherical harmonics orders $l_{\max }=$ $5,7,9,11$ to reconstruct the magnetic maps. For all epochs of our analysis except 2010.55 and 2015.54 , the target $\chi^{2}$ was achieved for $l_{\max }=7$. Using $l_{\max } \geq 7$ does not improve the $\chi^{2}$ or the fit to the spectra at epochs 2007.59, 2008.64, 2013.61, and 2014.61. However, for epochs 2010.55 and 2015.54 , the best $\chi^{2}$ fit was obtained for $l_{\max } \geq 11$. To make comparisons between the epochs we kept the $l_{\max }$ to 11 throughout our analysis. We also include differential rotation in the large-scale field reconstructions (see Sect. 5.3 for more details). 

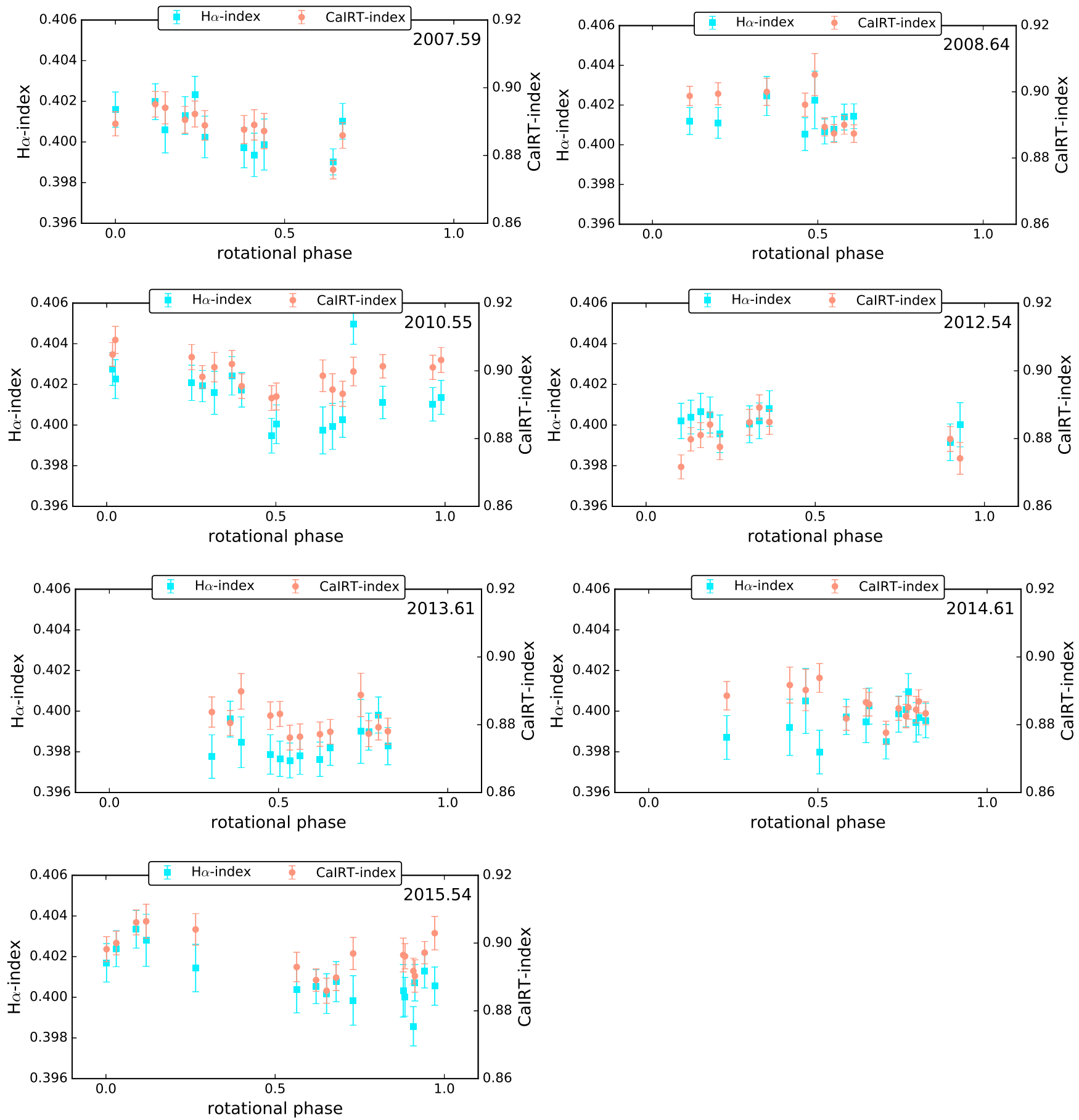

Fig. 3. Same as Fig. 1 for $\mathrm{H} \alpha$-index (left $y$-axis) and CaIRT-index (right $y$-axis). The scales are chosen based on the minimum and maximum value.

Figure 6 shows the Stokes $V$ LSD time series of 61 Cyg A for our observational time span. The fit between the modelled Stokes $V$ profiles and observed Stokes $V$ profiles was obtained with a reduced $\chi^{2}$ of 1.0 for epoch 2007.59. A reduced $\chi^{2}$ of 1.1 was achieved for epochs 2008.64, 2013.61, and 2014.61. For epochs 2010.55 and 2015.54 the observed fit was obtained with a reduced $\chi^{2}$ of 1.2 and 1.5 respectively. It is evident from Fig. 6 that during epoch 2007.59, which is around activity minimum, the time series of Stokes $V$ profiles is dominated by a single sign. The Stokes $V$ profiles in epoch 2010.55 are not dominated by a single orientation. The high variability shown by the Stokes $V$ profile in 2010.55 is caused by a complex magnetic field geometry. This complexity of the field is likely to make the impact of differential rotation more apparent in this epoch. In the following three epochs $(2013.61,2014.61$, and 2015.54) the Stokes $V$ profiles flip sign and are dominated again by a single orientation. This evolution of the Stokes $V$ time series indicates that the surface magnetic field geometry is strongly evolving, with a single polarity field during low activity and a more complex field during strong activity.

Since the maximum entropy reconstruction does not allow error calculations of our magnetic maps, we employ the technique described by Petit et al. (2008), where a range of magnetic maps were calculated for the wide range of input stellar 

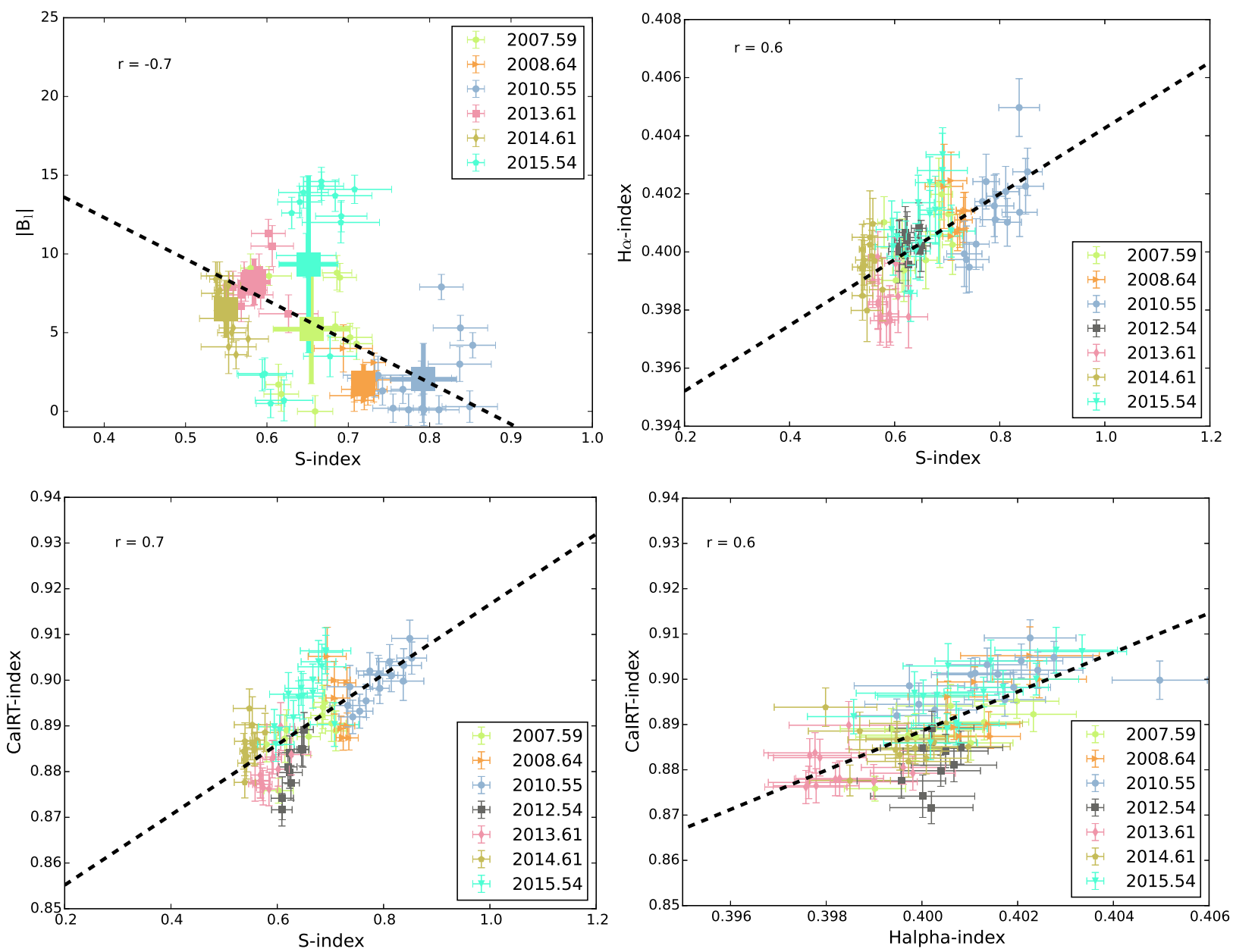

Fig. 4. Correlation between the different activity indicators and the longitudinal field $\left|B_{l}\right|$, where the dashed line represents least square fit to the data and the Pearson's $r$ is shown in the plot. Top left: anti-correlation between S-index and $\left|B_{l}\right|$ over six epochs of observations. The bold crosses represent the mean and standard deviation as dispersion. Only the mean values are included in the fit. Top right: plot showing the correlation between S-index and $\mathrm{H} \alpha$-index over seven epochs of observations. Bottom left: correlation between S-index and CaIRT-index over seven epochs of data. Bottom right: correlation between $\mathrm{H} \alpha$-index and CaIRT-index over seven epochs of data.

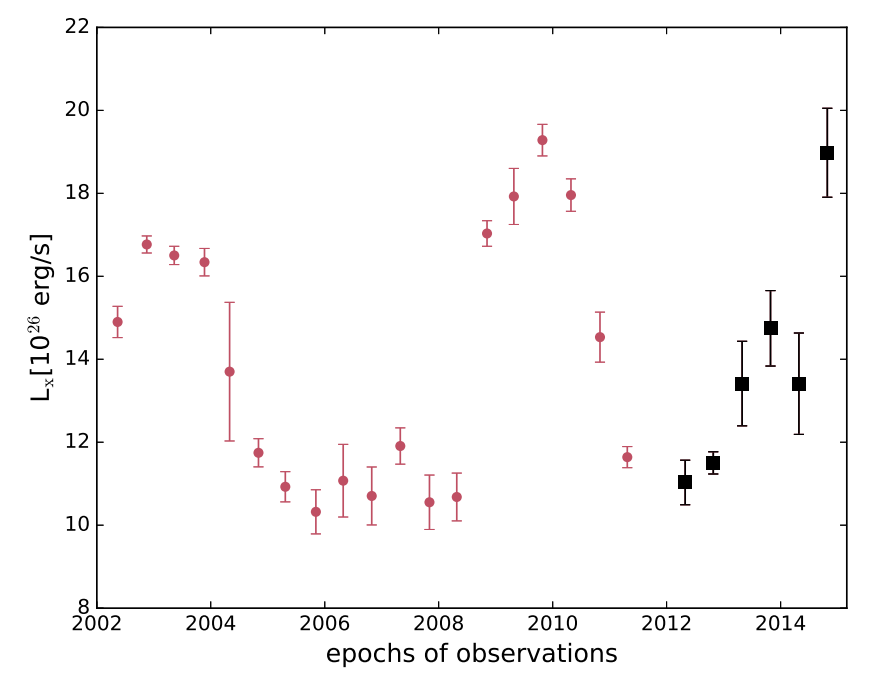

Fig. 5. Variation of X-ray luminosity of $61 \mathrm{Cyg}$ A determined through $X M M-N e w t o n$ observations. The black squares show new previously unpublished results.

parameters used in our ZDI code. These input parameters are varied within their error bars and the resulting dispersion is taken as error bars, as shown in Table 2.

\subsubsection{Epoch 2007.59}

The large-scale surface magnetic field of $61 \mathrm{Cyg}$ A at epoch 2007.59 exhibits a simple dipolar magnetic geometry. Figure 7 shows this large-scale magnetic field geometry reconstructed into three components: radial, azimuthal and meridional. The radial component of the magnetic field exhibits strong negativepolarity magnetic regions at higher latitudes and positivepolarity magnetic field at lower latitudes. On the other hand, the azimuthal field component is dominated by two positive polarity magnetic field regions between the pole and lower latitudes. The $B_{\text {mean }}$, which is the average of the surface magnetic field from the reconstructed maps, is $12 \pm 3 \mathrm{G}$. The poloidal magnetic field dominates, accounting for $93 \pm 5 \%$ of the magnetic energy seen in Stokes $V$. The fraction of the magnetic energy in other field components is shown in Table 2. As shown in Fig. 8, almost $100 \%$ of the poloidal magnetic energy is stored in the lower order spherical harmonics modes, $l \leq 3$.

\subsubsection{Epoch 2008.64}

In epoch 2008.64 the large-scale magnetic field in the radial component exhibits a similar magnetic geometry as reconstructed for epoch 2007.59, shown in Fig. 7. However, the mean magnetic field $\left(B_{\text {mean }}\right)$ is weaker than in the previous epoch, 
S. Boro Saikia et al.: 61 Cyg A

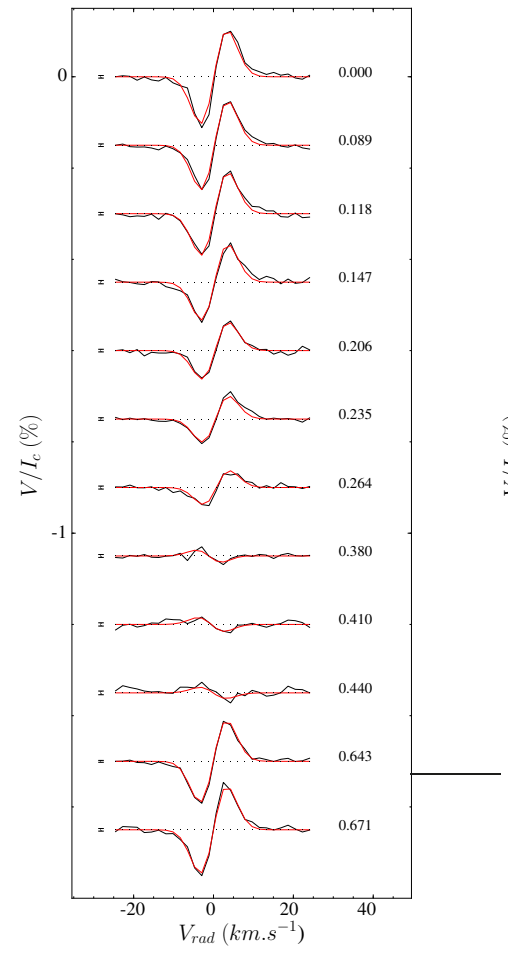

(a) 2007.59

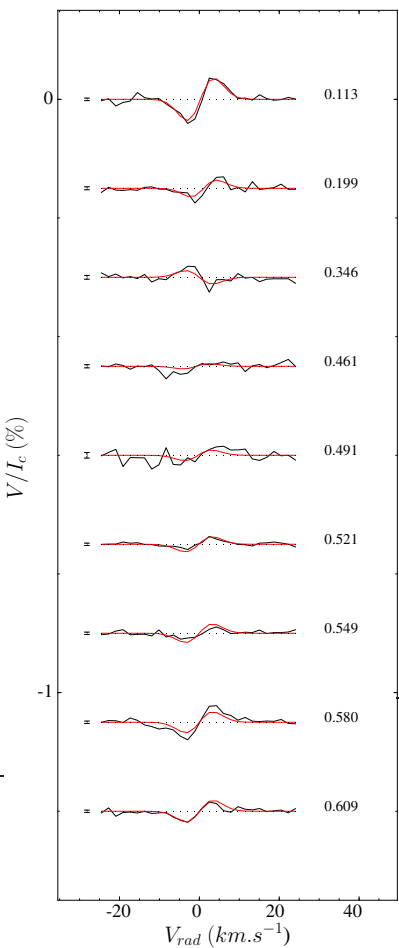

(b) 2008.64

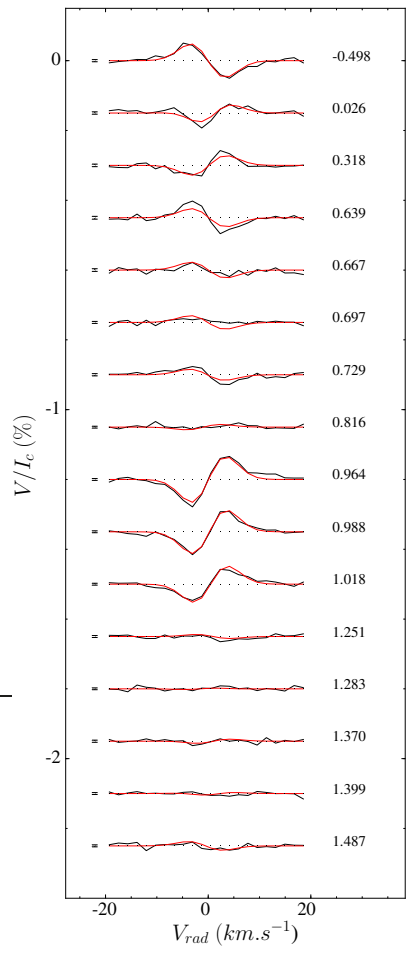

(c) 2010.55

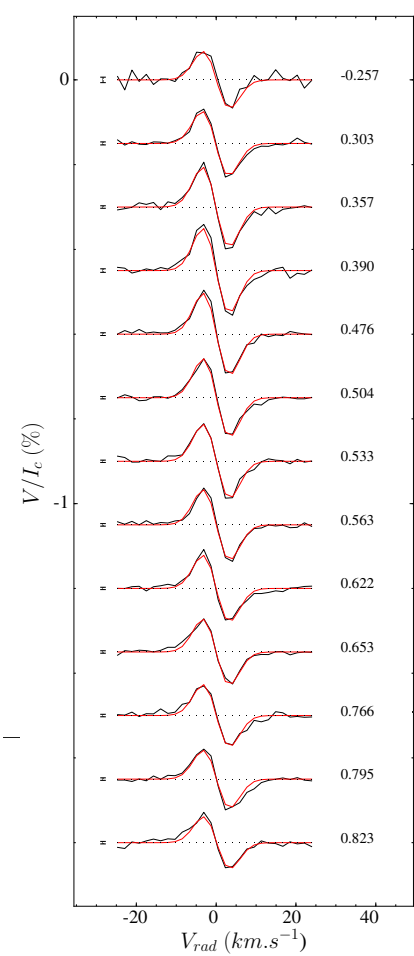

(d) 2013.61

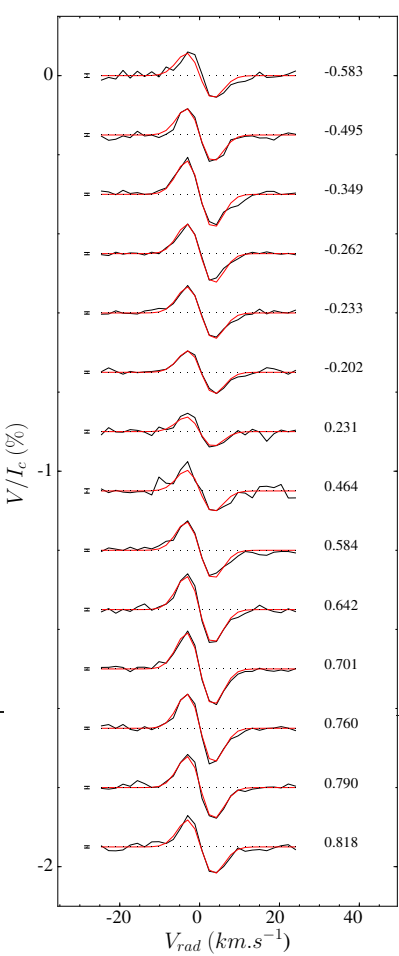

(e) 2014.61

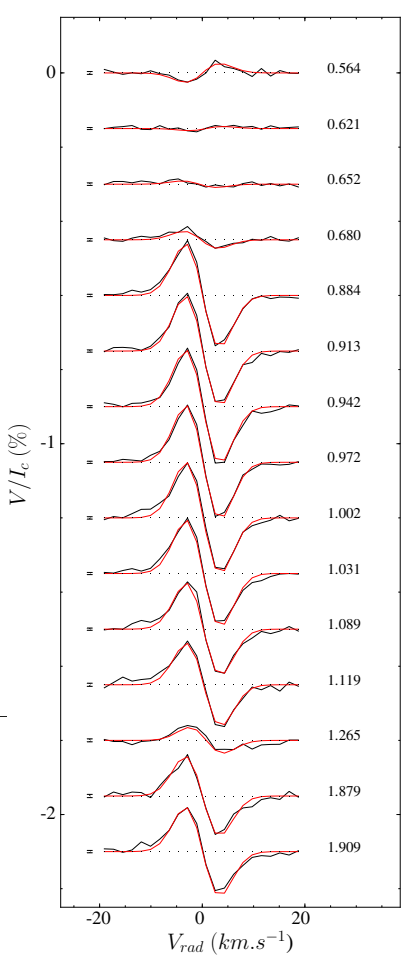

(f) 2015.54

Fig. 6. Time series of LSD Stokes $V$ spectra of 61 Cyg A, where top row (from left to right) represents epochs 2007.59, 2008.64, and 2010.55, and the bottom row shows (left to right) epochs 2013.61,2014.61, and 2015.54. The observed spectra is shown in black and the corresponding fit is shown in red. For each observed spectra, the phase of the rotational cycle is shown on the right, and on the left side of each plot $1 \sigma$ error is shown. For the purpose of clarity successive Stokes $V$ profiles are shifted vertically and the scale is expanded by a factor of two. 

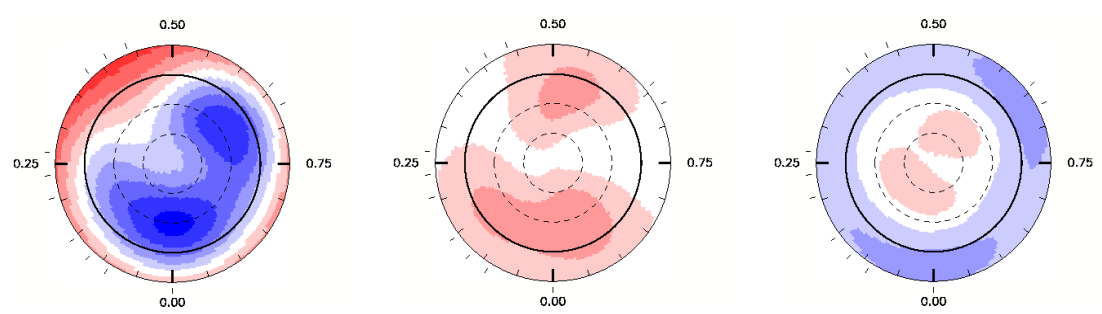

2008.64
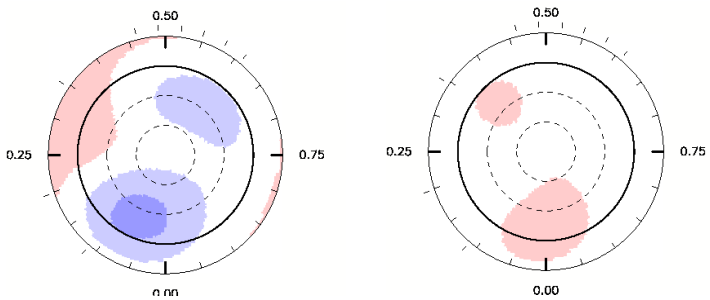

2010.55
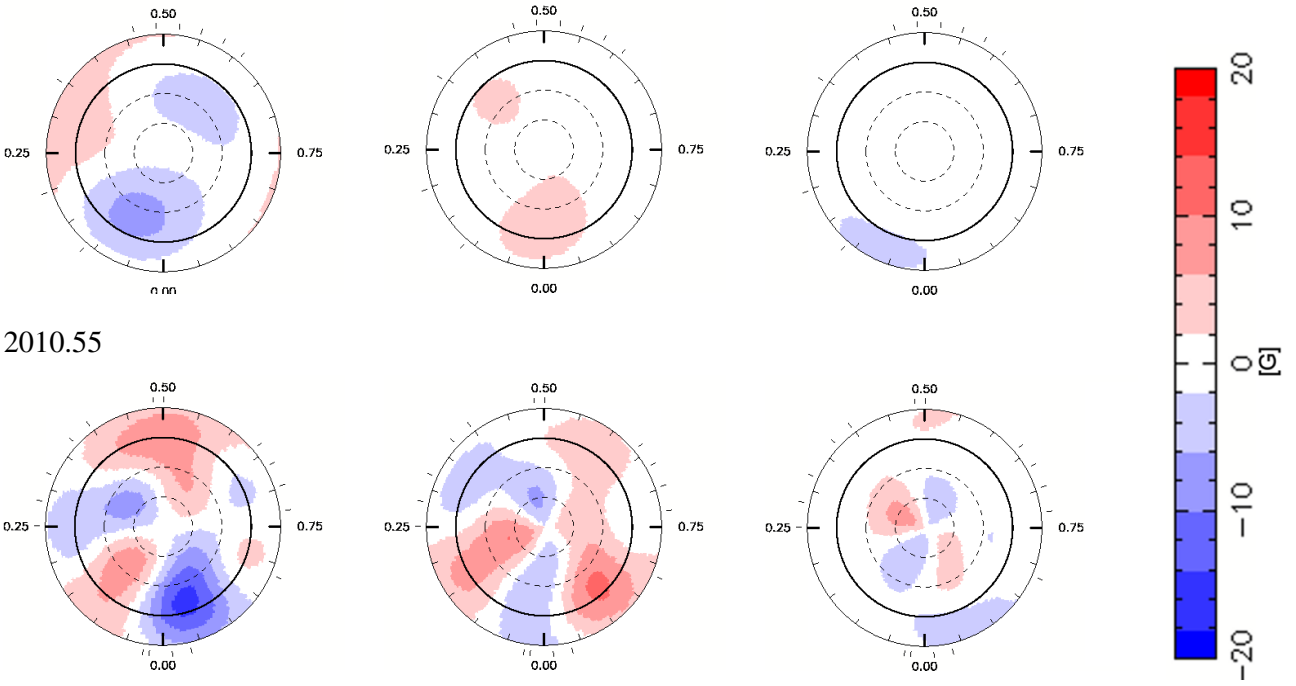

2013.61
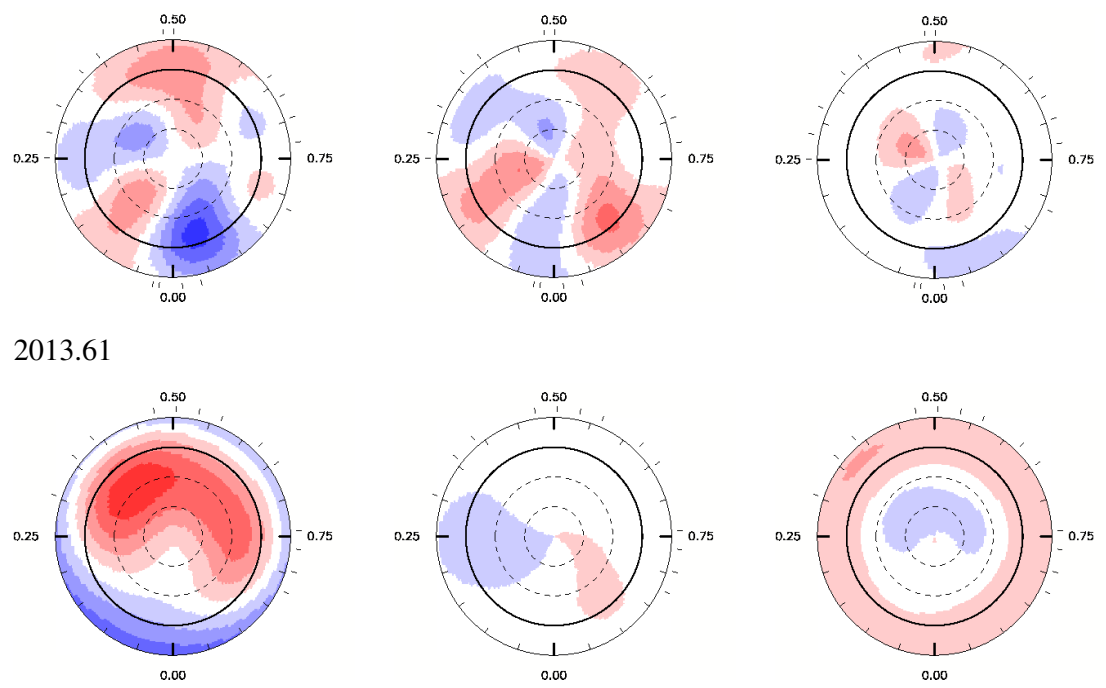

2014.61
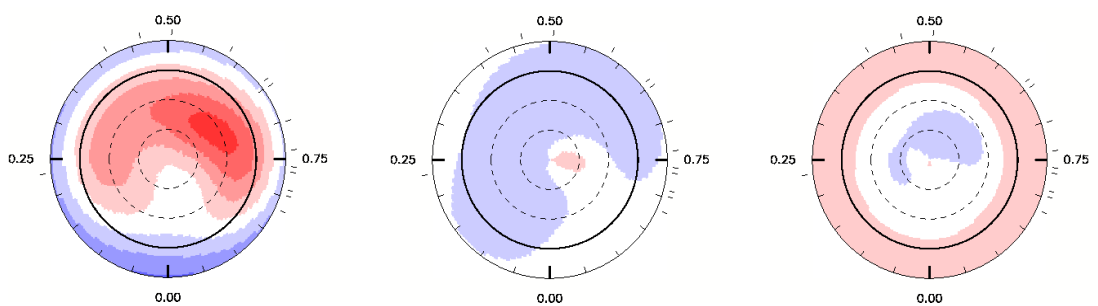

2015.54
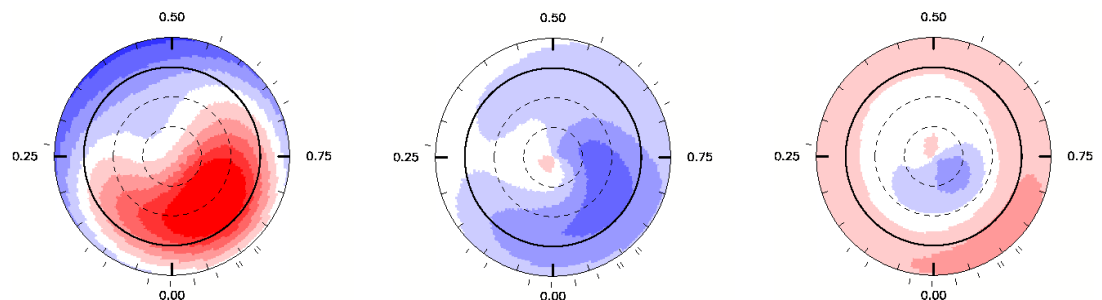

Fig. 7. Surface magnetic geometry of 61 Cyg A over six observational epochs $(2007.59,2008.64,2010.55,2013.61,2014.61$, and 2015.54). The three columns represent the radial, azimuthal, and the meridional component of the large-scale field. The star is shown in flattened polar projection down to latitudes of $-30^{\circ}$, where the dark line depicts the stellar equator. 
Table 2. Magnetic properties of 61 Cyg A extracted from the ZDI maps.

\begin{tabular}{|c|c|c|c|c|c|c|c|c|c|c|c|}
\hline Dates & $\begin{array}{c}\text { No of } \\
\text { obs }\end{array}$ & $\begin{array}{c}v_{r} \\
\left(\mathrm{~km} \mathrm{~s}^{-1}\right)\end{array}$ & $\begin{array}{c}B_{\text {mean }} \\
(\mathrm{G})\end{array}$ & $\begin{array}{c}\text { Poloidal } \\
\text { (\%tot) }\end{array}$ & $\begin{array}{l}\text { Dipole } \\
(\% \text { pol })\end{array}$ & $\begin{array}{l}\text { Quad } \\
\text { (\%pol) }\end{array}$ & $\begin{array}{c}\text { Oct } \\
(\% \text { pol })\end{array}$ & $\begin{array}{c}\text { Axi } \\
\text { (\%tot) }\end{array}$ & $\begin{array}{c}\text { Axi } \\
(\% \text { pol })\end{array}$ & $\begin{array}{c}\Omega_{\mathrm{eq}} \\
\left(\mathrm{rad} \mathrm{d}^{-1}\right)\end{array}$ & $\begin{array}{c}\mathrm{d} \Omega \\
\left(\operatorname{rad~d}^{-1}\right)\end{array}$ \\
\hline 2007.59 & 12 & $-65.47 \pm 0.05$ & $12 \pm 3$ & $93 \pm 5$ & $77 \pm 9$ & $17 \pm 7$ & $5 \pm 3$ & $80 \pm 13$ & $80 \pm 17$ & & \\
\hline 2008.64 & 9 & $-65.48 \pm 0.05$ & $3 \pm 1$ & $92 \pm 2$ & $56 \pm 5$ & $36 \pm 5$ & $8 \pm 2$ & $56 \pm 10$ & $53 \pm 11$ & & \\
\hline 2010.55 & 16 & $-65.48 \pm 0.05$ & $5 \pm 2$ & $87 \pm 3$ & & $21 \pm 5$ & $44 \pm 13$ & $7 \pm 2$ & $2 \pm 4$ & $0.18 \pm 0.03$ & $0.04 \pm 0.02$ \\
\hline 2013.61 & 13 & $-65.49 \pm 0.05$ & $9 \pm 6$ & $99_{-3}^{+1}$ & $80 \pm 9$ & $16 \pm 7$ & $3 \pm 1$ & $77 \pm 6$ & $77 \pm 6$ & & \\
\hline 2014.61 & 14 & $-65.50 \pm 0.05$ & $8 \pm 4$ & $93 \pm 4$ & $78 \pm 8$ & $18 \pm 6$ & $4 \pm 3$ & $81 \pm 7$ & $81 \pm 9$ & & \\
\hline 2015.54 & 15 & $-65.52 \pm 0.05$ & $12 \pm 5$ & $87 \pm 5$ & $85 \pm 9$ & $12 \pm 6$ & $2 \pm 1$ & $59 \pm 12$ & $55 \pm 20$ & & \\
\hline
\end{tabular}

Notes. The columns represent fractional dates, number of observations, radial velocity $\left(v_{\mathrm{r}}\right)$, mean magnetic field strength $\left(B_{\text {mean }}\right)$, fraction of magnetic energy reconstructed as the poloidal component, and fraction of poloidal magnetic field stored as dipole, quadrupole, and octopole modes. Finally it also shows the fraction of the total magnetic energy in the axisymmetric component of the magnetic field, and the poloidal axisymmetric fraction (for $m \leq \frac{l}{2}$ ). The differential rotation parameters $\Omega_{\mathrm{eq}}$ and $\mathrm{d} \Omega$ are also shown in the last two columns.

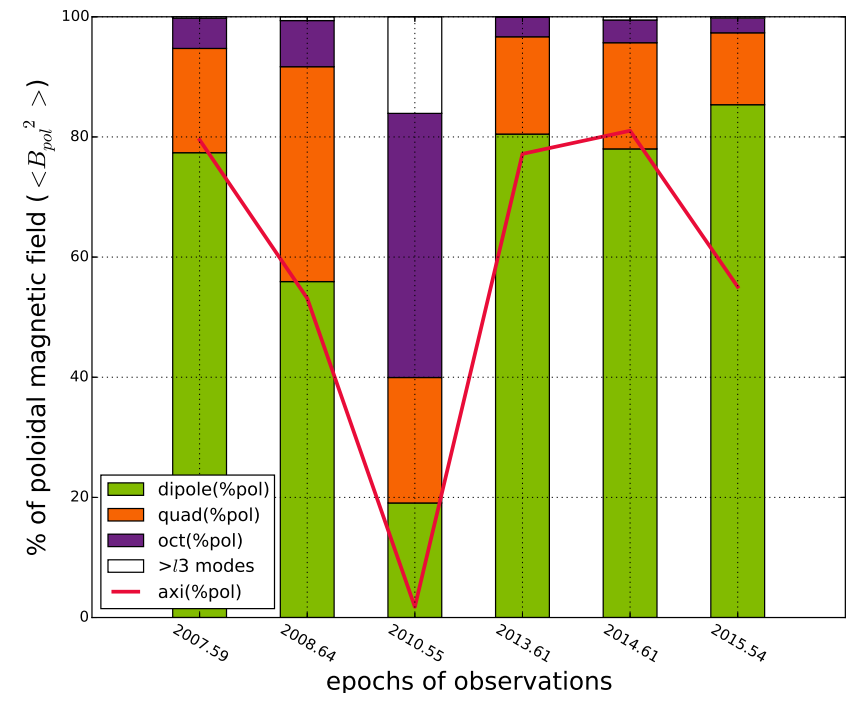

Fig. 8. Poloidal magnetic field distribution of $61 \mathrm{Cyg}$ A for six epoch of observations.

as shown in Table 2, where $B_{\text {mean }}$ is $3 \pm 1 \mathrm{G}$. In the azimuthal field component the positive polarity magnetic regions seen in the previous epoch are almost non-existent, as shown in Fig. 7. The magnetic field is predominantly poloidal, constituting $92 \pm 2 \%$ of the magnetic energy. The dipolar component of the poloidal field accounts for $56 \pm 5 \%$ of the poloidal magnetic energy. As in the previous epoch, the lower order modes $(l \leq 3)$ account for almost $100 \%$ of the poloidal magnetic energy budget as shown in Fig. 8.

\subsubsection{Epoch 2010.55}

The large-scale magnetic field of 61 Cyg A changes dramatically in epoch 2010.55. The high-latitude negative polarity field reconstructed in epoch 2007.59 is absent from this epoch and the radial field component is dominated by a more complex magnetic geometry between the equator and the poles, as shown in Fig. 7. The azimuthal field component also exhibits a more complex geometry compared to previous epochs. The averaged $B_{\text {mean }}$ for epoch 2010.55 is $5 \pm 2 \mathrm{G}$, which is still weaker than epoch 2007.59, as shown in Table 2. The poloidal field dominates and constitutes $87 \pm 3 \%$ of the magnetic energy. Only $19 \pm 6 \%$ of the poloidal field is dipolar, $21 \pm 5 \%$ is quadrupolar and $44 \pm 13 \%$ of the poloidal field is reconstructed into its octopolar component, as shown in Table 2. The field is also the least axisymmetric in this epoch. Unlike previous epochs, a few \% of the poloidal magnetic field is also distributed in the higher order spherical harmonics modes, as shown in Fig. 8. The percentage of the magnetic field reconstructed into its toroidal component is higher at this epoch when compared to the previous epochs.

\subsubsection{Epoch 2013.61}

The large-scale magnetic field geometry at epoch 2013.61 is shown in Fig. 7, where higher latitude magnetic regions are reconstructed in the radial field component with opposite polarity, as seen in epochs 2007.59 and 2008.64. The azimuthal field component exhibits mixed polarity but it is weak and almost nonexistent, as shown in Fig. 7. The mean magnetic field derived from the magnetic maps $\left(B_{\text {mean }}=9 \pm 6 \mathrm{G}\right)$ is stronger than the previous two epochs. The poloidal component comprises $99_{-3}^{+1} \%$ of the magnetic energy, as shown in Table 2. The poloidal magnetic field is $80 \pm 9 \%$ dipolar. The distribution of the magnetic field is primarily concentrated at the lower spherical harmonics modes (dipolar, quadrupolar, and octopolar) as shown in Fig. 8.

\subsubsection{Epoch 2014.61}

The strong positive field in epoch 2013.61 is also reconstructed in the radial component in epoch 2014.61, as shown in Fig. 7. The azimuthal field, as shown in Fig. 7, is reconstructed as a band of negative-polarity magnetic field at the surface of the star, where the field is of opposite polarity, as seen in epoch 2007.59. Table 2 shows the mean magnetic field $B_{\text {mean }}$ of epoch 2014.61, where the field strength is $8 \pm 4 \mathrm{G}$. The fraction of the magnetic field distributed into the different field components is shown in Table 2, where $93 \pm 4 \%$ of the magnetic field is reconstructed into its poloidal component. $78 \pm 8 \%$ of the poloidal field is in its dipolar component. The magnetic energy budget is spread across the lower spherical harmonics mode $l \leq 3$, as shown in Fig. 8 .

\subsubsection{Epoch 2015.54}

During epoch 2015.54 the radial field component exhibits a positive polarity magnetic region between the poles and the equator, together with a negative polarity magnetic field at the equator, as shown in Fig. 7. A band of negative polarity magnetic field is reconstructed in the azimuthal component of the magnetic field, as shown in Fig. 7. The mean magnetic field $B_{\text {mean }}$ of $12 \pm 5 \mathrm{G}$ is similar to that measured in epoch 2007.59 . $87 \pm 5 \%$ of the 


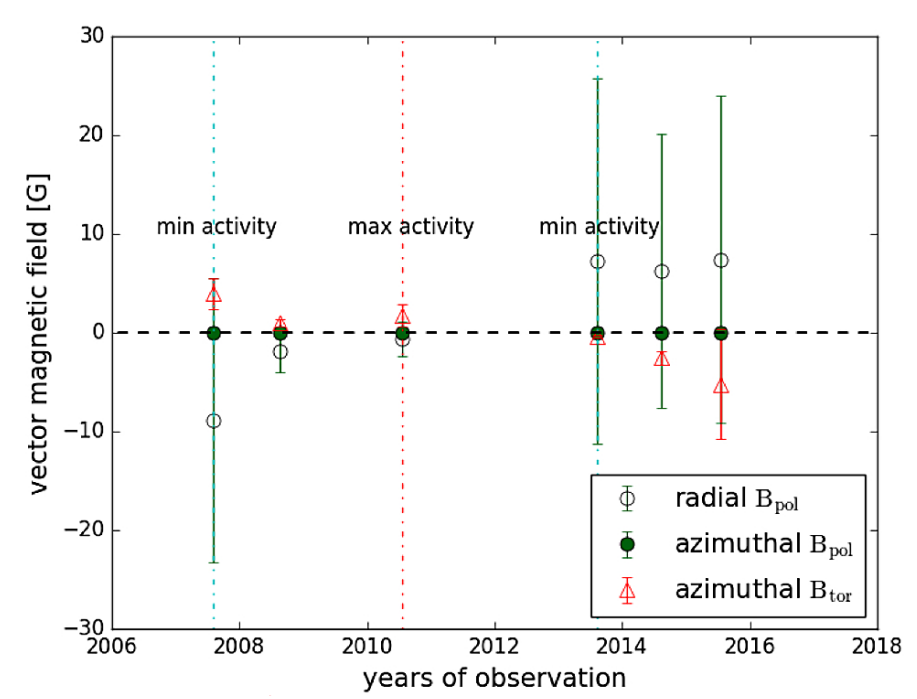

Fig. 9. Vector component of the poloidal and toroidal magnetic field strength averaged over the stellar surface. The field strengths in the radial and azimuthal components are shown. The horizontal dashed line shows where the field strength is 0 . The cyan vertical dashed lines show the epochs with minimum activity and the red vertical dashed line shows the epoch with maximum chromospheric activity.

magnetic field is in the poloidal component, as shown in Table 2. The percentage of the poloidal field reconstructed into the dipolar component is $85 \pm 9 \%$ and, as shown in Fig. 8 the poloidal field is distributed across the lower spherical harmonics modes.

\subsection{Evolution of the different multipolar modes over the magnetic cycle}

In order to assess the long-term variability of the magnetic field of $61 \mathrm{Cyg} \mathrm{A}$, we consider several ways of paraphrasing the reconstructed magnetic field. First, we measure the vector component of the magnetic field for each epoch, averaged over the stellar surface. The vector radial field component, as mentioned earlier in this section, is entirely poloidal in nature and the signed value of this component is shown in Fig. 9. The field is averaged over the stellar surface for co-latitudes less than 30 , which is close to the rotational pole. Here co-latitudes are surface co-latitudes relative to the stellar rotational pole in spherical co-ordinates. The vector azimuthal field, on the other hand, is comprised of both poloidal and toroidal field components in this case. Therefore, the signed azimuthal poloidal field and the signed azimuthal toroidal field are treated separately and calculated by averaging the surface for co-latitudes less than 30 . As seen in Fig. 9, the poloidal field strength $B_{\text {pol }}$ in the radial component is weakest in epoch 2010.55 and is stronger during epochs of low chromospheric activity. The poloidal component of the azimuthal field is almost negligible for the total azimuthal field. From Fig. 9 it is clear that the poloidal field strength on the stellar surface, for co-latitudes $\leq 30^{\circ}$, is mostly radial. The toroidal field strength $B_{\text {tor }}$ exhibits weak anti correlation to the radial field strength $B_{\text {pol }}$.

Since the axisymmetric modes are of greater interest for existing dynamo models, we investigate the time evolution of both even and odd axisymmetric modes $(m=0)$ for $l=1,2,3,4$. Figure 10 shows the field strength, at the pole of the axisymmetric modes of the poloidal field, for the first four orders of spherical harmonics. The poloidal field strength in the axisymmetric modes is the signed (radial) field strength at the visible rotational pole of the star. The first odd mode $l=1$, which is the

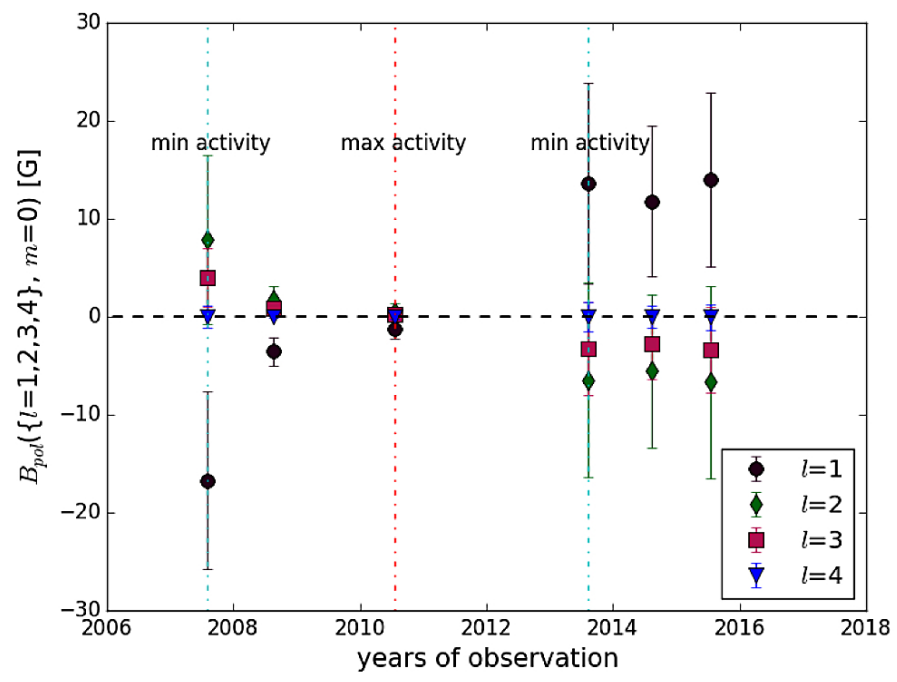

Fig. 10. Magnetic field strength $B_{\mathrm{r}}$ at the magnetic pole for the different axisymmetric modes $(l=\{1,2,3\})$. The horizontal dashed line represents $B_{\mathrm{r}}=0$, and the vertical dashed lines represents the epochs with minimum and maximum activity as shown in Fig. 9.

dipolar mode, exhibits similar behaviour to the full averaged $B_{\mathrm{pol}}$ in Fig. 9. The quadrupolar $l=2$ mode is anti-correlated with the dipolar mode. The octopolar mode $(l=3)$ exhibits correlation with the quadrupolar mode.

Figure 11 shows the field strength of only the lowest order $(l=1)$ axisymmetric $(m=0)$ poloidal and toroidal modes. The poloidal field strength is the same as that shown in Fig. 10, but only the dipolar mode is plotted. The evolution of the dipolar field is consistent with the polarity reversals of the large-scale radial field shown in Fig. 7 and shows strong correlation with the averaged radial poloidal field strength in Fig. 9. The signed toroidal field strength is calculated by measuring the strength of the azimuthal component of the toroidal $(l=0, m=0)$ field, evaluated at the maximum of the toroidal band. The toroidal field strength is anti-correlated with the poloidal field strength in Fig. 11.

The uncertainties were estimated using the same technique as described in Sect. 5.1.

\subsection{Differential rotation}

Each epoch spanned over multiple weeks; we therefore investigated the effect of differential rotation on our data. In order to investigate the differential rotation parameters of $61 \mathrm{Cyg} \mathrm{A}$, we incorporated a simple solar-type differential rotation law into our ZDI code, as described by Petit et al. (2002),

$\Omega(l)=\Omega_{\mathrm{eq}}-\mathrm{d} \Omega \sin ^{2} l$

where $\Omega(l)$ represents the stellar rotation at latitude $l, \Omega_{\mathrm{eq}}$ is the equatorial rotation rate, and $\mathrm{d} \Omega$ is the difference between the rotation rate at the equator and the poles.

We set the magnetic field strength to a fixed value and carry out ZDI reconstruction for a wide range of differential rotation parameters $\Omega_{\mathrm{eq}}$ and $\mathrm{d} \Omega$ in a $2 \mathrm{D}$ parameter space. The set of differential rotation parameters for which the $\chi^{2}$ minimum is achieved is then selected, as shown in Fig. 12. The uncertainties were calculated by the bootstrap technique, where the input stellar parameters were varied within their error bars and the differential rotation was measured for this wide range of input parameters. The resulting dispersion in the obtained values is considered as the associated uncertainties and is shown in Table 2. 


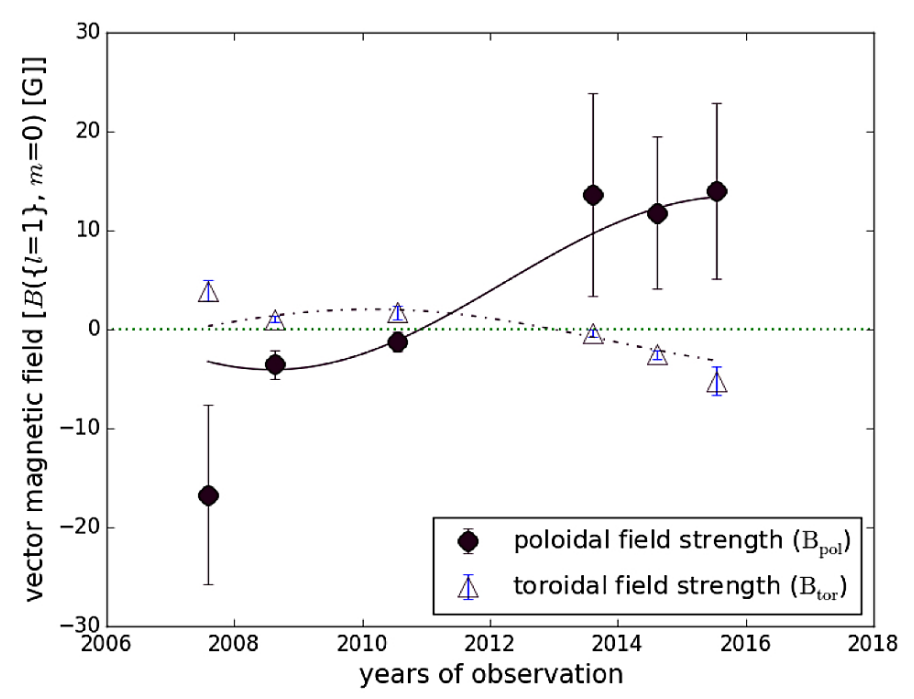

Fig. 11. Magnetic field strength $B$ at the magnetic pole for the axisymmetric $l=1$ mode for the poloidal component, which is similar to $l=1$ in Fig. 10. For the toroidal component, $B$ represents the strength of the latitudinal or azimuthal field. The green horizontal dashed line represents $B=0$. The fits are obtained with a magnetic cycle period of $14.4 \mathrm{yr}$ which is twice the activity cycle period of $7.2 \mathrm{yr}$.

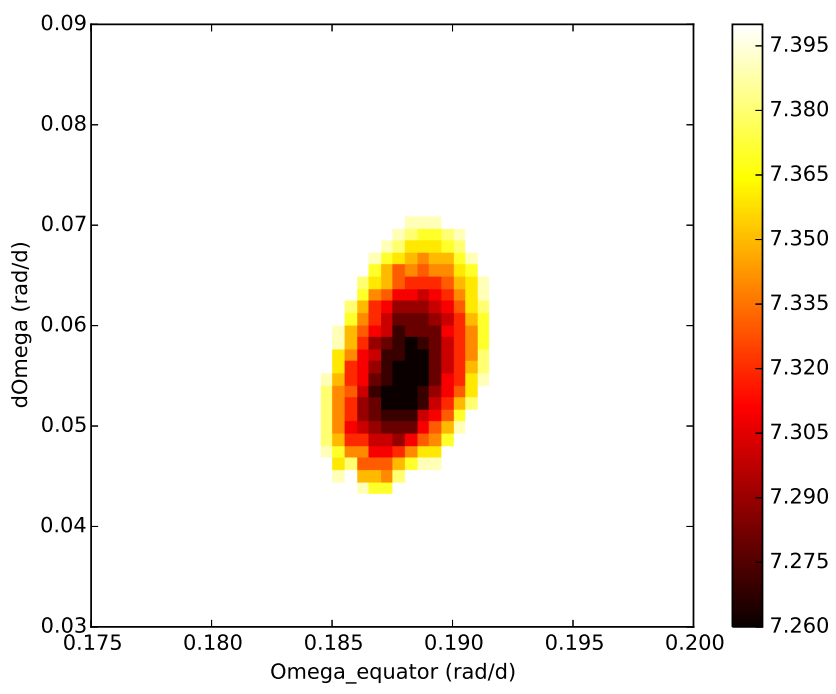

Fig. 12. Best fit $B_{\text {mean }}$ map obtained by varying the differential parameters for epoch 2010.55 . The $\Omega_{\mathrm{eq}}$ and $\mathrm{d} \Omega$ values obtained from this map are $0.184 \mathrm{rad} \mathrm{d}^{-1}$ and $0.04 \mathrm{rad} \mathrm{d}^{-1}$ respectively.

To obtain reliable differential rotation parameters, it is necessary to have observations with good phase coverage spread over multiple rotation periods of the star (Petit et al. 2002; Morgenthaler et al. 2012). Consequently, the differential parameters for 61 Cyg A could only be obtained for epoch 2010.55, as shown in Table 2. For epoch 2010.55 we measured a $\Omega_{\mathrm{eq}}=$ $0.18 \mathrm{rad} \mathrm{d}^{-1}$ and $\mathrm{d} \Omega=0.04 \mathrm{rad} \mathrm{d}^{-1}$. We obtained a rotation period $P_{\text {rot }}$ of $34.2 \pm 3.7$ days. This rotation period is in agreement with the rotation period obtained from chromospheric measurements, as shown in Table 1 . Using this $\mathrm{d} \Omega$ and $\Omega$ value we determine the relative horizontal shear $(\alpha)$ of 0.2 which is equivalent to the $\alpha_{\odot}=0.2$.

Since the phase coverage of the other epochs was sparse, we could not obtain differential rotation measurements as reliable as those in epoch 2010.55. The magnetic field geometry is also simple with single polarity dominating the surface in all epochs except 2010.55 making it harder to determine the differential rotation for those epochs. Hence, we adopted the differential rotation parameters of 2010.55 for the rest of the observational epochs.

\section{Long-term evolution of the magnetic and activity cycle}

The long-term evolution of the chromospheric indicators and the magnetic field strength is shown in Fig. 13. The average value of the different chromospheric activity indicators exhibits good correlation over a long timescale. Figure 13 also shows the mean longitudinal magnetic field of $61 \mathrm{Cyg} \mathrm{A}$ averaged over each epoch, where the error bars represent the standard deviation in $B_{l}$ per observational epoch. Strong temporal variations are detected for the average longitudinal field, with stronger field strength in epochs 2007.59, 2013.61, 2014.61 and 2015.54. The field strength is the weakest in epochs 2008.64 and 2010.55. However, we note that epochs 2007.59 and 2010.55 exhibit stronger dispersion in $B_{l}$ compared to the other epochs. Long-term observations of $61 \mathrm{Cyg}$ A exhibits a weak anti-correlation between the mean magnetic field strength and the different chromospheric activity indicators.

The mean magnetic field obtained from the reconstructed ZDI maps averaged over each epoch is also shown in Fig. 13. $B_{\text {mean }}$ follows a similar trend to $B_{l}$; however, $B_{\text {mean }}$ is weakest in epoch 2008.64 and does not show any pronounced anticorrelation with the $\mathrm{S}$-index.

\section{Discussion}

The large-scale magnetic field geometry of the solar-type K5 dwarf 61 Cyg A was reconstructed using ZDI for five observational epoch spread over nine years, covering a complete chromospheric activity cycle. It exhibits clear polarity reversals of its large-scale magnetic field geometry.

\subsection{Large-scale magnetic field}

\subsubsection{Polarity reversal of the large-scale field}

We report polarity reversals of the large-scale magnetic field of 61 Cyg A, where, for the first time, a strong correlation is detected between the magnetic cycle and the chromospheric activity cycle. Polarity reversals have been previously detected in the large-scale magnetic field reconstructions of several cool stars such as $\tau$ Boo, HD 190771, and HD 78366. However, the polarity reversals detected in previous targets do not exhibit any correlation with the star's chromospheric activity cycle.

The $v \sin i$ of $61 \mathrm{Cyg} \mathrm{A}$ is lower than other ZDI targets. A previous numerical study by Kochukhov \& Piskunov (2002) has shown that although reconstructions of the surface geometry from spectropolarimetric data can be carried out for slow rotators, the resolution of the surface reconstruction decreases with lower vsini. Since $61 \mathrm{Cyg} \mathrm{A}$ is a slow rotator, we can only resolve down the surface elements into the lower order spherical harmonics modes. In spite of this shortcoming, the polarity reversal of 61 Cyg A is clearly seen in the Stokes $V$ spectra itself. Figure 6 clearly demonstrates that the Stokes $V$ profiles change with rotational phase and also change with the chromospheric activity cycle. As shown in Fig. 6, the form of the Stokes $V$ profile in epoch 2007.59 flips in epoch 2013.61, indicating that the 


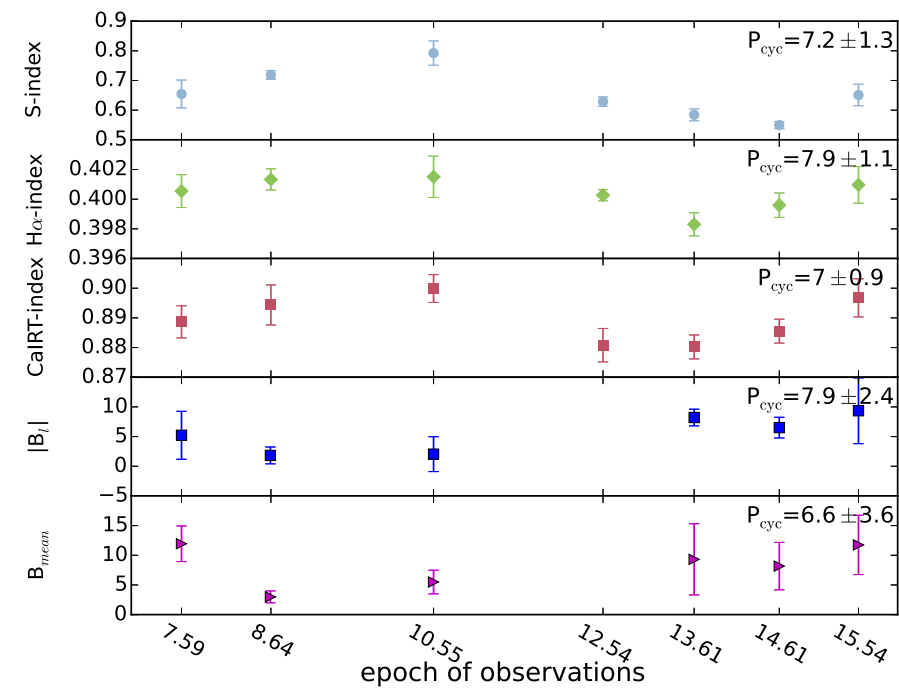

Fig. 13. Long-term evolution of the average values of the activity indices, the average longitudinal field, and the mean magnetic field measured from the ZDI maps.

large-scale field also flips polarity. The lack of any dominant Stokes $V$ shape in epoch 2010.55 also indicates that the largescale field is more complex during that epoch. The time series of Stokes $V$ profiles reconfirms that the polarity reversals of the large-scale field are in fact independent of any artefact.

\subsubsection{Evolution of the multipolar modes of the large-scale field}

The magnetic field of 61 Cyg A's large-scale field is strongly poloidal at all epochs of our observations, which we quantify through energy fractions $\left(\left\langle B^{2}\right\rangle\right)$. Particularly at epoch 2013.61, which is the epoch around which the field geometry flips polarity, the poloidal magnetic field constitutes $99 \%$ of the total magnetic energy budget as seen in Stokes $V$. During epoch 2010.55 which is coincidentally the chromospheric activity maximum, the percentage of the toroidal magnetic field increases in comparison to other epochs. The axisymmetry of the poloidal field component also reaches its minimum in epoch 2010.55, as shown in Fig. 8. It indicates that the field is complex during activity maximum, in a similar way to the Sun. The poloidal field is dominated by the dipolar component except during activity maximum, when the other higher order modes dominate.

We investigate the lower order spherical harmonics modes of the star and interpret these results in the context of the Sun. Throughout our observational time span, the vector magnetic field, averaged over the surface, is stronger in the poloidal component except during epoch 2010.55 . During activity maximum the vector component of the both the poloidal and toroidal field averaged over the surface of the star is close to zero. During epochs of minimum activity, we detected a weak anti-correlation between the poloidal and toroidal field.

The low order axisymmetric modes of the poloidal field indicate that the dipolar component is strongest compared to the quadrupolar and octopolar modes throughout our observational time span. The poloidal dipolar component $(l=1)$ is strongest during epochs of low activity and is almost negligible during activity maximum in epoch 2010.55 . The other higher order modes are almost negligible throughout our observations. The largescale solar magnetic field of the Sun is also more dipolar during epochs of low activity (DeRosa et al. 2012). The quadrupolar component of the solar large-scale field dominates during activity maximum which is not seen in $61 \mathrm{Cyg} \mathrm{A}$. One explanation for this disparity between the modes of 61 Cyg A might be caused by cross-talk between the odd and even sets of modes. To investigate that we suppressed the even modes and measured the field coefficients again. Although the odd modes are favoured over the even modes, no improvements were detected. An alternative explanation might be that the ZDI reconstruction technique has different sensitivities to the different spherical harmonics.

\subsection{Chromospheric activity}

The chromospheric activity of $61 \mathrm{Cyg}$ A was determined by using three activity proxies: the $\mathrm{Ca}$ II $\mathrm{H} \& \mathrm{~K}, \mathrm{H} \alpha$, and $\mathrm{Ca}$ II IRT lines. From the combined S-index data, we measure a cycle period of $7.2 \pm 1.3 \mathrm{yr}$. An activity cycle period of $7.3 \pm 0.1 \mathrm{yr}$ was also previously determined by the long-term Mount Wilson survey (Baliunas et al. 1995).

A correlation is detected between the S-index and $\mathrm{H} \alpha$-index of $61 \mathrm{Cyg} \mathrm{A}$ as shown in Fig. 4. Such correlation is also observed in the Sun, where the correlation between the two indices follows the activity cycle (Livingston et al. 2007). Correlations were also observed for other BCool targets such as $\xi$ Boo A (Morgenthaler et al. 2012) and HN Peg (Boro Saikia et al. 2015). The correlation between the fluxes in Ca II H\&K lines and the $\mathrm{H} \alpha$ lines was investigated by Cincunegui et al. (2007) for 109 cool stars. They observed strong dispersion in correlations with both positive and negative correlations. They also suggested that the correlation does not depend on activity and spectral type but on stellar colour.

The correlation between a star's S-index and H $\alpha$-index has been further studied by Meunier \& Delfosse (2009), who suggest that one of the main factors affecting the correlation of these two indices is the filling factor of plages and filaments on the stellar surfaces. Since plages affect both $\mathrm{Ca}$ II $\& \mathrm{~K}$ and $\mathrm{H} \alpha$ lines, and filaments are mostly observed as absorption in $\mathrm{H} \alpha$, they claim that the correlations depended strongly on the spatial and temporal distribution of filaments on the stellar surface. They also observe an activity dependence on the correlations, where stronger correlation was observed near the cycle maxima. They also suggest that the correlations might also depend on time span of the observations and stellar inclination.

A correlation was also observed between 61 Cyg A's S-index and IRT-index and between $\mathrm{H} \alpha$-index and IRT-index (both shown in Fig. 4). A stronger correlation is observed between the S-index and the IRT-index. This is not surprising as the Ca II IRT lines also arise from plage dominated areas on the stellar surface (Andretta et al. 2005; Busà et al. 2007). In the BCool snapshot study Marsden et al. (2014) also reports a good correlation between these two indices.

A weak anti-correlation was observed between the S-index and the longitudinal magnetic field. In previous ZDI targets no correlation was observed between the chromospheric activity and longitudinal magnetic field $B_{1}$, for example, in $\xi$ Boo A (Morgenthaler et al. 2012) and HN Peg (Boro Saikia et al. 2015). The lack of correlation is expected due to the cancellation of small scale magnetic features in the mean field measurements. However, for $61 \mathrm{Cyg}$ A the longitudinal magnetic field $B_{1}$ is weaker during activity maximum and stronger during activity minimum. No anti-correlation was detected, however, between the chromospheric S-index and the mean magnetic field $\left(B_{\text {mean }}\right)$ measured from the magnetic maps. The chromospheric activity of 61 Cyg A shows good agreement with its coronal activity cycle which is also seen in the Sun. 


\section{Summary}

In this paper we reconstruct the large-scale surface geometry of $61 \mathrm{Cyg}$ A for six epochs over nine years of observations. We report the presence of a possible magnetic cycle which is twice the length of the activity cycle. This is the first detection of a cool star, apart from the Sun, where the magnetic cycle is in phase with its chromospheric cycle. The large-scale surface magnetic field geometry of 61 Cyg A flips its polarity and is highly variable, with the radial field showing strong polar field during activity minimum and a complex field during activity maximum. Throughout our observations, the large-scale field of $61 \mathrm{Cyg}$ A is strongly poloidal except during activity minimum when the poloidal field is relatively weaker. The dipolar component of the poloidal field is strongest close to activity minimum and is weakest during activity maximum. During activity maximum, higher order modes such as quadrupolar and octopolar modes dominate over the dipolar mode. The evolution of the large-scale field of 61 Cyg A over the activity cycle shows close resemblance to the solar large-scale field, which has never been seen before in cool stars.

Acknowledgements. We thank Dr. Jan Robrade of Hamburger Sternwarte for his help in data analysis of the XMM-Newton data. This work was carried out as part of Project A16 funded by the Deutsche Forschungsgemeinschaft (DFG) under SFB 963. AAV acknowledges support from an Ambizione Fellowship of the Swiss National Science Foundation. C.P.F. was supported by the grant ANR 2011 Blanc SIMI5-6 02001 "Toupies: Towards understanding the spin evolution of stars". Part of the work was also supported by the COST action MP1104 "Polarisation as a tool to study the Solar-system and beyond". Finally, we thank the anonymous referee for their detailed and constructive comments.

\section{References}

Andretta, V., Busà, I., Gomez, M. T., \& Terranegra, L. 2005, A\&A, 430, 669 Aurière, M. 2003, EAS Pub. Ser., 9, 105

Ayres, T. R. 2015, AJ, 149, 58

Baliunas, S. L., Donahue, R. A., Soon, W. H., et al. 1995, ApJ, 438, 269

Barnes, S. A. 2007, ApJ, 669, 1167

Bessel, F. W. 1838, MNRAS, 4, 152

Boro Saikia, S., Jeffers, S. V., Petit, P., et al. 2015, A\&A, 573, A17

Busà, I., Aznar Cuadrado, R., Terranegra, L., Andretta, V., \& Gomez, M. T. 2007, A\&A, 466, 1089

Cincunegui, C., Díaz, R. F., \& Mauas, P. J. D. 2007, A\&A, 469, 309

DeRosa, M. L., Brun, A. S., \& Hoeksema, J. T. 2012, ApJ, 757, 96

Donati, J.-F. 2003, in Solar Polarization, eds. J. Trujillo-Bueno, \& J. Sanchez Almeida, ASP Conf. Ser., 307, 41

Donati, J.-F., Semel, M., Carter, B. D., Rees, D. E., \& Collier Cameron, A. 1997, MNRAS, 291, 658
Donati, J.-F., Howarth, I. D., Jardine, M. M., et al. 2006, MNRAS, 370,629 Donati, J.-F., Moutou, C., Farès, R., et al. 2008, MNRAS, 385, 1179 Donati, J.-F., Gregory, S. G., Alencar, S. H. P., et al. 2012, MNRAS, 425, 2948 Duncan, D. K., Vaughan, A. H., Wilson, O. C., et al. 1991, ApJS, 76, 383

Fares, R., Donati, J.-F., Moutou, C., et al. 2009, MNRAS, 398, 1383 Fares, R., Moutou, C., Donati, J.-F., et al. 2013, MNRAS, 435, 1451 Favata, F., Micela, G., Orlando, S., et al. 2008, A\&A, 490, 1121 Gizis, J. E., Reid, I. N., \& Hawley, S. L. 2002, AJ, 123, 3356 Güdel, M. 2004, A\&ARv, 12, 71

Hall, J. C. 2008, Liv. Rev. Sol. Phys., 5, 2

Hall, J. C., Lockwood, G. W., \& Skiff, B. A. 2007, AJ, 133, 862 Hathaway, D. H. 2010, Liv. Rev. Sol. Phys., 7, 1

Hempelmann, A., Schmitt, J. H. M. M., \& Stępień, K. 1996, A\&A, 305, 284 Hempelmann, A., Robrade, J., Schmitt, J. H. M. M., et al. 2006, A\&A, 460, 261 Jefferies, J. T., \& Thomas, R. N. 1959, ApJ, 129, 401

Jeffers, S. V., Petit, P., Marsden, S. C., et al. 2014, A\&A, 569, A79

Kervella, P., Mérand, A., Pichon, B., et al. 2008, A\&A, 488, 667

Kochukhov, O., \& Piskunov, N. 2002, A\&A, 388, 868

Kochukhov, O., Makaganiuk, V., \& Piskunov, N. 2010, A\&A, 524, A5

Livingston, W., Wallace, L., White, O. R., \& Giampapa, M. S. 2007, ApJ, 657, 1137

Lockwood, G. W., Skiff, B. A., Henry, G. W., et al. 2007, ApJS, 171, 260

Lomb, N. R. 1976, Ap\&SS, 39, 447

Malkov, O. Y., Tamazian, V. S., Docobo, J. A., \& Chulkov, D. A. 2012, A\&A, 546, A69

Mamajek, E. E., \& Hillenbrand, L. A. 2008, ApJ, 687, 1264

Mann, A. W., Gaidos, E., \& Ansdell, M. 2013, ApJ, 779, 188

Marsden, S. C., Petit, P., Jeffers, S. V., et al. 2014, MNRAS, 444, 3517

Mengel, M. W., Fares, R., Marsden, S. C., et al. 2016, MNRAS, 459, 4325

Meunier, N., \& Delfosse, X. 2009, A\&A, 501, 1103

Morgenthaler, A., Petit, P., Morin, J., et al. 2011, Astron. Nachr., 332, 866

Morgenthaler, A., Petit, P., Saar, S., et al. 2012, A\&A, 540, A138

Perryman, M. A. C., Lindegren, L., Kovalevsky, J., et al. 1997, A\&A, 323, L49

Petit, P., Donati, J.-F., \& Collier Cameron, A. 2002, MNRAS, 334, 374

Petit, P., Dintrans, B., Solanki, S. K., et al. 2008, MNRAS, 388, 80

Petit, P., Dintrans, B., Morgenthaler, A., et al. 2009, A\&A, 508, L9

Pevtsov, A. A., Fisher, G. H., Acton, L. W., et al. 2003, ApJ, 598, 1387

Reiners, A., \& Basri, G. 2006, ApJ, 644, 497

Robinson, Jr., R. D. 1980, ApJ, 239, 961

Robrade, J., Schmitt, J. H. M. M., \& Favata, F. 2012, A\&A, 543, A84

Saar, S. H. 1996, in Stellar Surface Structure, eds. K. G. Strassmeier, \& J. L. Linsky, IAU Symp., 176, 237

Sanderson, T. R., Appourchaux, T., Hoeksema, J. T., \& Harvey, K. L. 2003, J. Geophys. Res. (Space Physics), 108, 1035

Sanz-Forcada, J., Stelzer, B., \& Metcalfe, T. S. 2013, A\&A, 553, L6

Scargle, J. D. 1982, ApJ, 263, 835

Schröder, K.-P., Mittag, M., Hempelmann, A., González-Pérez, J. N., \& Schmitt, J. H. M. M. 2013, A\&A, 554, A50

Semel, M. 1989, A\&A, 225, 456

Skilling, J., \& Bryan, R. K. 1984, MNRAS, 211, 111

Takeda, G., Ford, E. B., Sills, A., et al. 2007, ApJS, 168, 297

Valenti, J. A., \& Fischer, D. A. 2005, ApJS, 159, 141

Wilson, O. C. 1978, ApJ, 226, 379

Wright, J. T., Marcy, G. W., Butler, R. P., \& Vogt, S. S. 2004, ApJS, 152, 261

Zechmeister, M., \& Kürster, M. 2009, A\&A, 496, 577 
A\&A 594, A29 (2016)

\section{Appendix A: Additional tables}

Table A.1. Journal of observations for seven epochs (2007.59, 2008.64, 2010.55, 2012.54, 2013.61, 2014.61, and 2015.54).

\begin{tabular}{|c|c|c|c|c|}
\hline Date & $\begin{array}{c}\text { Julian date } \\
(2450000+)\end{array}$ & $\begin{array}{c}\text { Exposure time } \\
\text { (s) }\end{array}$ & $S / N$ & $\begin{array}{c}\sigma_{\mathrm{LSD}} \\
\left(10^{-5} I_{\mathrm{c}}\right)\end{array}$ \\
\hline 2007 July 26 & 4308.49809 & 1200 & 35765 & 2.7960 \\
\hline 2007 July 30 & 4312.52989 & 800 & 35807 & 2.7928 \\
\hline 2007 July 31 & 4313.53121 & 800 & 27096 & 3.6905 \\
\hline 2007 August 02 & 4315.53608 & 800 & 33873 & 2.9522 \\
\hline 2007 August 03 & 4316.53315 & 800 & 35102 & 2.8489 \\
\hline 2007 August 04 & 4317.53462 & 800 & 30572 & 3.2710 \\
\hline 2007 August 08 & 4321.50738 & 800 & 31550 & 3.1696 \\
\hline 2007 August 09 & 4322.52760 & 800 & 28744 & 3.4790 \\
\hline 2007 August 10 & 4323.52773 & 800 & 24362 & 4.1048 \\
\hline 2007 August 17 & 4330.50089 & 1600 & 48802 & 2.0491 \\
\hline 2007 August 18 & 4331.46147 & 800 & 35561 & 2.8121 \\
\hline 2008 August 09 & 4688.54994 & 1200 & 46210 & 2.1641 \\
\hline 2008 August 12 & 4691.48653 & 1200 & 40079 & 2.4951 \\
\hline 2008 August 17 & 4696.52126 & 1200 & 31218 & 3.2033 \\
\hline 2008 August 21 & 4700.47785 & 1200 & 36751 & 2.7210 \\
\hline 2008 August 22 & 4701.48433 & 1200 & 21527 & 4.6454 \\
\hline 2008 August 23 & 4702.50793 & 1200 & 50016 & 1.999 \\
\hline 2008 August 24 & 4703.47966 & 1200 & 49602 & 2.0161 \\
\hline 2008 August 25 & 4704.53225 & 1200 & 48263 & 2.0720 \\
\hline 2008 August 26 & 4705.52953 & 1200 & 49904 & 2.0039 \\
\hline 2010 June 03 & 5351.64786 & 900 & 32082 & 3.1171 \\
\hline 2010 June 21 & 5369.57210 & 900 & 31021 & 3.2236 \\
\hline 2010 July 01 & 5379.58460 & 900 & 27037 & 3.6987 \\
\hline 2010 July 12 & 5390.54209 & 900 & 25343 & 3.9458 \\
\hline 2010 July 13 & 5391.52388 & 900 & 26541 & 3.7678 \\
\hline 2010 July 14 & 5392.54747 & 900 & 34955 & 2.8608 \\
\hline 2010 July 15 & 5393.63958 & 900 & 31139 & 3.2114 \\
\hline 2010 July 18 & 5396.60809 & 900 & 38824 & 2.5757 \\
\hline 2010 July 23 & 5401.65920 & 900 & 37562 & 2.6623 \\
\hline 2010 July 24 & 5402.49800 & 900 & 37471 & 2.6687 \\
\hline 2010 July 25 & 5403.49549 & 900 & 37945 & 2.6354 \\
\hline 2010 August 02 & 5411.49161 & 900 & 35432 & 2.8224 \\
\hline 2010 August 03 & 5412.56953 & 900 & 40189 & 2.4883 \\
\hline 2010 August 06 & 5415.55556 & 900 & 32469 & 3.0799 \\
\hline 2010 August 07 & 5416.54320 & 900 & 36301 & 2.7548 \\
\hline 2010 August 10 & 5419.56875 & 900 & 36220 & 2.7610 \\
\hline 2012 July 08 & 6117.63676 & 900 & 33129 &.. \\
\hline 2012 July 09 & 6118.63476 & 900 & 28811 & .. \\
\hline 2012 July 15 & 6124.64113 & 900 & 35051 & .. \\
\hline 2012 July 16 & 6125.61827 & 900 & 36914 & .. \\
\hline 2012 July 17 & 6126.61222 & 900 & 34327 & .. \\
\hline 2012 July 18 & 6127.56778 & 900 & 34221 & .. \\
\hline 2012 July 19 & 6128.55829 & 900 & 32787 & .. \\
\hline 2012 July 22 & 6131.55361 & 900 & 34150 & .. \\
\hline 2012 July 23 & 6132.54263 & 900 & 35066 & .. \\
\hline 2012 July 24 & 6133.56659 & 900 & 34955 &.. \\
\hline 2013 July 14 & 6488.51321 & 800 & 15411 & 6.4889 \\
\hline 2013 August 02 & 6507.65889 & 800 & 28131 & 3.5548 \\
\hline 2013 August 04 & 6509.51420 & 800 & 34901 & 2.8653 \\
\hline 2013 August 05 & 6510.61746 & 800 & 24113 & 4.1472 \\
\hline 2013 August 08 & 6513.57114 & 800 & 31163 & 3.2090 \\
\hline 2013 August 09 & 6514.54087 & 800 & 35398 & 2.8251 \\
\hline 2013 August 10 & 6515.54442 & 800 & 35718 & 2.7997 \\
\hline 2013 August 11 & 6516.56402 & 800 & 33745 & 2.9634 \\
\hline 2013 August 13 & 6518.58190 & 800 & 36075 & 2.7720 \\
\hline
\end{tabular}

Notes. Column 1 represents the year and date of observations, Col. 2 is the Julian date, col. 3 is the exposure time, Col. 4 is the signal-to-noise ratio of each Stokes $V$ LSD profile, and Col. 5 represents the error bars in the Stokes $V$ LSD profile. 
Table A.1. continued.

\begin{tabular}{|c|c|c|c|c|}
\hline Date & $\begin{array}{c}\text { Julian date } \\
(2450000+)\end{array}$ & $\begin{array}{c}\text { Exposure time } \\
\text { (s) }\end{array}$ & $S / N$ & $\begin{array}{c}\sigma_{\mathrm{LSD}} \\
\left(10^{-5} I_{\mathrm{c}}\right)\end{array}$ \\
\hline 2013 August 14 & 6519.62914 & 800 & 35479 & 2.8186 \\
\hline 2013 August 18 & 6523.51294 & 800 & 33442 & 2.9903 \\
\hline 2013 August 19 & 6524.49914 & 800 & 34439 & 2.9036 \\
\hline 2013 August 20 & 6525.44457 & 800 & 33347 & 2.9988 \\
\hline 2014 July 14 & 6853.55493 & 800 & 21642 & 4.6207 \\
\hline 2014 July 17 & 6856.55743 & 800 & 28094 & 3.5596 \\
\hline 2014 July 22 & 6861.57410 & 800 & 34595 & 2.8906 \\
\hline 2014 July 25 & 6864.55546 & 800 & 34374 & 2.9092 \\
\hline 2014 July 26 & 6865.53839 & 800 & 35150 & 2.8449 \\
\hline 2014 July 27 & 6866.58350 & 800 & 35645 & 2.8055 \\
\hline 2014 August 11 & 6881.38890 & 800 & 27850 & 3.5907 \\
\hline 2014 August 19 & 6889.36216 & 800 & 18535 & 5.3954 \\
\hline 2014 August 23 & 6893.46397 & 800 & 35628 & 2.8068 \\
\hline 2014 August 25 & 6895.46092 & 800 & 29424 & 3.3987 \\
\hline 2014 August 27 & 6897.47963 & 800 & 36306 & 2.7544 \\
\hline 2014 August 29 & 6899.49512 & 800 & 35859 & 2.7888 \\
\hline 2014 August 30 & 6900.52575 & 800 & 31814 & 3.1433 \\
\hline 2014 August 31 & 6901.48870 & 800 & 36408 & 2.7467 \\
\hline 2015 June 26 & 7200.57599 & 900 & 26403 & 3.7874 \\
\hline 2015 June 28 & 7202.53874 & 900 & 36708 & 2.7242 \\
\hline 2015 June 29 & 7203.59939 & 900 & 30539 & 3.2745 \\
\hline 2015 June 30 & 7204.56304 & 900 & 30669 & 3.2607 \\
\hline 2015 July 07 & 7211.52277 & 900 & 31832 & 3.1415 \\
\hline 2015 July 08 & 7212.52460 & 900 & 34962 & 2.8603 \\
\hline 2015 July 09 & 7213.50574 & 900 & 37205 & 2.6878 \\
\hline 2015 July 10 & 7214.52800 & 900 & 33875 & 2.9521 \\
\hline 2015 July 11 & 7215.56071 & 900 & 32176 & 3.1080 \\
\hline 2015 July 12 & 716.54418 & 900 & 34501 & 2.8985 \\
\hline 2015 July 14 & 7218.55681 & 900 & 32888 & 3.0407 \\
\hline 2015 July 15 & 7219.56343 & 900 & 23679 & 4.2233 \\
\hline 2015 July 20 & 7224.56050 & 900 & 25336 & 3.9469 \\
\hline 2015 August 05 & 7240.48208 & 900 & 24468 & 4.0871 \\
\hline 2015 August 10 & 7245.54843 & 900 & 22931 & 4.3610 \\
\hline 2015 August 11 & 7246.57034 & 900 & 31250 & 3.2000 \\
\hline
\end{tabular}


Table A.2. The chromospheric activity and longitudinal field measurements of 61 Cyg A for six epochs $(2007.59,2008.64,2010.55,2012.54$, 2013.61, 2014.61, and 2015.54).

\begin{tabular}{|c|c|c|c|c|c|c|c|}
\hline Epoch & $\begin{array}{c}\text { Julian date } \\
(2454000+)\end{array}$ & Rot. phase & S-index & $\mathrm{H} \alpha$-index & CaIRT-index & $\begin{array}{c}B_{l} \\
(\mathrm{G}) \\
\end{array}$ & $\begin{array}{c}N_{l} \\
(\mathrm{G}) \\
\end{array}$ \\
\hline \multirow{11}{*}{2007.59} & 4308.49809 & 0.00000 & $0.69 \pm 0.02$ & $0.402 \pm 0.001$ & $0.889 \pm 0.003$ & $-10.0 \pm 0.9$ & $-0.8 \pm 0.9$ \\
\hline & 4312.52989 & 0.11789 & $0.69 \pm 0.02$ & $0.402 \pm 0.001$ & $0.895 \pm 0.003$ & $-8.5 \pm 0.9$ & $-0.0 \pm 0.9$ \\
\hline & 4313.53121 & 0.14717 & $0.69 \pm 0.01$ & $0.401 \pm 0.001$ & $0.894 \pm 0.004$ & $-8.8 \pm 1.1$ & $-1.1 \pm 1.1$ \\
\hline & 4315.53608 & 0.20579 & $0.70 \pm 0.02$ & $0.401 \pm 0.001$ & $0.891 \pm 0.003$ & $-4.7 \pm 0.9$ & $0.1 \pm 0.9$ \\
\hline & 4316.53315 & 0.23494 & $0.68 \pm 0.02$ & $0.402 \pm 0.001$ & $0.892 \pm 0.003$ & $-5.4 \pm 0.9$ & $0.1 \pm 0.9$ \\
\hline & 4317.53462 & 0.26423 & $0.71 \pm 0.01$ & $0.400 \pm 0.001$ & $0.889 \pm 0.004$ & $-4.3 \pm 1.0$ & $0.6 \pm 1.0$ \\
\hline & 4321.50738 & 0.38039 & $0.66 \pm 0.02$ & $0.399 \pm 0.001$ & $0.888 \pm 0.004$ & $0.0 \pm 1.0$ & $-0.2 \pm 1.0$ \\
\hline & 4322.52760 & 0.41022 & $0.62 \pm 0.02$ & $0.399 \pm 0.001$ & $0.889 \pm 0.004$ & $1.1 \pm 1.1$ & $-0.6 \pm 1.1$ \\
\hline & 4323.52773 & 0.43946 & $0.61 \pm 0.01$ & $0.400 \pm 0.001$ & $0.887 \pm 0.005$ & $1.7 \pm 1.3$ & $0.6 \pm 1.3$ \\
\hline & 4330.50089 & 0.64336 & $0.60 \pm 0.02$ & $0.399 \pm 0.001$ & $0.876 \pm 0.002$ & $-8.6 \pm 0.6$ & $-0.4 \pm 0.6$ \\
\hline & 4331.46147 & 0.67144 & $0.58 \pm 0.01$ & $0.401 \pm 0.001$ & $0.886 \pm 0.003$ & $-9.1 \pm 0.9$ & $0.4 \pm 0.9$ \\
\hline \multirow{9}{*}{2008.64} & 4688.54994 & 0.11263 & $0.73 \pm 0.01$ & $0.401 \pm 0.001$ & $0.898 \pm 0.003$ & $-2.9 \pm 0.7$ & $0.3 \pm 0.7$ \\
\hline & 4691.48653 & 0.19849 & $0.73 \pm 0.02$ & $0.401 \pm 0.001$ & $0.899 \pm 0.003$ & $-1.4 \pm 0.8$ & $-0.9 \pm 0.8$ \\
\hline & 4696.52126 & 0.34571 & $0.71 \pm 0.03$ & $0.402 \pm 0.001$ & $0.900 \pm 0.004$ & $1.0 \pm 1.0$ & $0.4 \pm 1.0$ \\
\hline & 4700.47785 & 0.46140 & $0.71 \pm 0.02$ & $0.400 \pm 0.001$ & $0.896 \pm 0.004$ & $-1.4 \pm 0.8$ & $0.2 \pm 0.8$ \\
\hline & 4701.48433 & 0.49083 & $0.69 \pm 0.04$ & $0.402 \pm 0.002$ & $0.905 \pm 0.006$ & $-4.0 \pm 1.5$ & $-0.3 \pm 1.5$ \\
\hline & 4702.50793 & 0.52076 & $0.72 \pm 0.01$ & $0.400 \pm 0.001$ & $0.889 \pm 0.003$ & $-0.7 \pm 0.6$ & $0.4 \pm 0.6$ \\
\hline & 4703.47966 & 0.54917 & $0.72 \pm 0.01$ & $0.401 \pm 0.001$ & $0.887 \pm 0.003$ & $-1.0 \pm 0.6$ & $1.1 \pm 0.6$ \\
\hline & 4704.53225 & 0.57995 & $0.73 \pm 0.01$ & $0.401 \pm 0.001$ & $0.890 \pm 0.003$ & $-3.1 \pm 0.6$ & $-0.1 \pm 0.6$ \\
\hline & 4705.52953 & 0.60911 & $0.73 \pm 0.02$ & $0.401 \pm 0.001$ & $0.887 \pm 0.003$ & $-2.0 \pm 0.6$ & $0.2 \pm 0.6$ \\
\hline \multirow{16}{*}{2010.55} & & & & & & & \\
\hline & 5369.57210 & 0.02556 & $0.85 \pm 0.03$ & $0.402 \pm 0.001$ & $0.909 \pm 0.004$ & $0.3 \pm 1.0$ & $-0.6 \pm 1.0$ \\
\hline & 5379.58460 & 0.31832 & $0.79 \pm 0.04$ & $0.402 \pm 0.001$ & $0.901 \pm 0.004$ & $-2.1 \pm 1.1$ & $-1.9 \pm 1.1$ \\
\hline & 5390.54209 & 0.63871 & $0.74 \pm 0.04$ & $0.400 \pm 0.001$ & $0.899 \pm 0.005$ & $2.3 \pm 1.2$ & $1.4 \pm 1.2$ \\
\hline & 5391.52388 & 0.66742 & $0.73 \pm 0.03$ & $0.399 \pm 0.001$ & $0.894 \pm 0.005$ & $2.1 \pm 1.2$ & $1.6 \pm 1.2$ \\
\hline & 5392.54747 & 0.69735 & $0.76 \pm 0.02$ & $0.400 \pm 0.001$ & $0.893 \pm 0.004$ & $0.2 \pm 0.9$ & $1.0 \pm 0.9$ \\
\hline & 5393.63958 & 0.72928 & $0.84 \pm 0.04$ & $0.405 \pm 0.001$ & $0.900 \pm 0.004$ & $3.0 \pm 1.1$ & $-0.8 \pm 1.1$ \\
\hline & 5396.60809 & 0.81608 & $0.79 \pm 0.03$ & $0.401 \pm 0.001$ & $0.901 \pm 0.003$ & $-0.2 \pm 0.9$ & $-0.7 \pm 0.9$ \\
\hline & 5401.65920 & 0.96378 & $0.81 \pm 0.03$ & $0.401 \pm 0.001$ & $0.901 \pm 0.004$ & $-7.9 \pm 0.8$ & $0.2 \pm 0.8$ \\
\hline & 5402.49800 & & & $0.401 \pm 0.001$ & $0.903 \pm 0.004$ & $-5.3 \pm 0.8$ & $-0.3 \pm 0.8$ \\
\hline & 5403.49549 & 0.01747 & $0.85 \pm 0.03$ & $0.403 \pm 0.001$ & $0.905 \pm 0.004$ & $-4.2 \pm 0.8$ & $0.5 \pm 0.8$ \\
\hline & 5411.49161 & 0.25127 & $0.81 \pm 0.02$ & $0.402 \pm 0.001$ & $0.904 \pm 0.004$ & $0.1 \pm 0.9$ & $-0.3 \pm 0.9$ \\
\hline & 5412.56953 & 0.28279 & $0.79 \pm 0.02$ & $0.402 \pm 0.001$ & $0.898 \pm 0.003$ & $0.1 \pm 1.0$ & $-0.6 \pm 1.0$ \\
\hline & 5415.55556 & 0.37010 & $0.77 \pm 0.03$ & $0.402 \pm 0.001$ & $0.902 \pm 0.004$ & $0.1 \pm 1.0$ & $-0.6 \pm 1.0$ \\
\hline & 5416.54320 & 0.39897 & $0.77 \pm 0.01$ & $0.402 \pm 0.001$ & $0.895 \pm 0.004$ & $1.4 \pm 0.9$ & $-1.5 \pm 0.9$ \\
\hline & 5419.56875 & 0.48745 & $0.74 \pm 0.03$ & $0.399 \pm 0.001$ & $0.892 \pm 0.004$ & $1.3 \pm 0.9$ & $0.1 \pm 0.9$ \\
\hline \multirow{10}{*}{2012.54} & 6117.63676 & 0.89879 & $0.61 \pm 0.02$ & $0.399 \pm 0.001$ & $0.880 \pm 0.004$ &.. & \\
\hline & 6118.63476 & 0.92797 & $0.61 \pm 0.02$ & $0.400 \pm 0.001$ & $0.874 \pm 0.005$ & .. & \\
\hline & 6124.64113 & 0.10360 & $0.61 \pm 0.02$ & $0.400 \pm 0.001$ & $0.872 \pm 0.004$ & .. & .. \\
\hline & 6125.61827 & 0.13217 & $0.62 \pm 0.02$ & $0.400 \pm 0.001$ & $0.880 \pm 0.003$ & .. & \\
\hline & 6126.61222 & 0.16123 & $0.62 \pm 0.02$ & $0.401 \pm 0.001$ & $0.881 \pm 0.004$ & .. & \\
\hline & 6127.56778 & 0.18917 & $0.62 \pm 0.02$ & $0.400 \pm 0.001$ & $0.884 \pm 0.004$ & .. & \\
\hline & 6128.55829 & 0.21813 & $0.63 \pm 0.02$ & $0.400 \pm 0.001$ & $0.878 \pm 0.004$ & .. & \\
\hline & 6131.55361 & 0.30572 & $0.65 \pm 0.01$ & $0.400 \pm 0.001$ & $0.885 \pm 0.004$ & .. & .. \\
\hline & 6132.54263 & 0.33464 & $0.65 \pm 0.02$ & $0.400 \pm 0.001$ & $0.889 \pm 0.004$ & .. & \\
\hline & 6133.56659 & 0.36458 & $0.65 \pm 0.01$ & $0.401 \pm 0.001$ & $0.885 \pm 0.004$ &.. &.. \\
\hline
\end{tabular}

Notes. From left to right it represents: Julian date, rotational phase, S-index, $\mathrm{H} \alpha$-index, CaIRT-index, longitudinal magnetic field ( $\left.B_{l}\right)$, and magnetic field of the Null profile $\left(N_{l}\right)$. The longitudinal magnetic field measurements are not included for 2012.54 epoch due to spectropolarimetric errors at NARVAL. See Sect. 3 for details. 
Table A.2. continued.

\begin{tabular}{|c|c|c|c|c|c|c|c|}
\hline Epoch & $\begin{array}{l}\text { Julian date } \\
(2454000+)\end{array}$ & Rot. phase & S-index & $\mathrm{H} \alpha$-index & CaIRT-index & $\begin{array}{c}B_{l} \\
(\mathrm{G})\end{array}$ & $\begin{array}{l}N_{l} \\
(\mathrm{G})\end{array}$ \\
\hline \multirow{13}{*}{2013.61} & 6488.51321 & 0.74313 & $0.62 \pm 0.06$ & $0.399 \pm 0.002$ & $0.889 \pm 0.006$ & $3.4 \pm 2.0$ & $-2.7 \pm 2.1$ \\
\hline & 6507.65889 & 0.30295 & $0.63 \pm 0.04$ & $0.398 \pm 0.001$ & $0.884 \pm 0.004$ & $6.2 \pm 1.2$ & $0.5 \pm 1.2$ \\
\hline & 6509.51420 & 0.35720 & $0.60 \pm 0.02$ & $0.400 \pm 0.001$ & $0.881 \pm 0.004$ & $11.3 \pm 0.9$ & $0.3 \pm 0.9$ \\
\hline & 6510.61746 & 0.38946 & $0.61 \pm 0.03$ & $0.398 \pm 0.001$ & $0.890 \pm 0.005$ & $10.5 \pm 1.3$ & $-0.2 \pm 1.3$ \\
\hline & 6513.57114 & 0.47582 & $0.59 \pm 0.02$ & $0.398 \pm 0.001$ & $0.883 \pm 0.004$ & $9.0 \pm 1.0$ & $-0.1 \pm 1.0$ \\
\hline & 6514.54087 & 0.50417 & $0.59 \pm 0.02$ & $0.398 \pm 0.001$ & $0.883 \pm 0.004$ & $7.4 \pm 0.9$ & $-0.0 \pm 0.9$ \\
\hline & 6515.54442 & 0.53352 & $0.58 \pm 0.02$ & $0.397 \pm 0.001$ & $0.876 \pm 0.004$ & $7.7 \pm 0.9$ & $-0.1 \pm 0.9$ \\
\hline & 6516.56402 & 0.56333 & $0.57 \pm 0.01$ & $0.398 \pm 0.001$ & $0.876 \pm 0.004$ & $7.9 \pm 0.9$ & $0.7 \pm 0.9$ \\
\hline & 6518.58190 & 0.62233 & $0.57 \pm 0.02$ & $0.398 \pm 0.001$ & $0.877 \pm 0.004$ & $8.6 \pm 0.9$ & $0.7 \pm 0.9$ \\
\hline & 6519.62914 & 0.65295 & $0.57 \pm 0.02$ & $0.398 \pm 0.001$ & $0.878 \pm 0.004$ & $7.9 \pm 0.9$ & $-0.2 \pm 0.9$ \\
\hline & 6523.51294 & 0.76652 & $0.56 \pm 0.01$ & $0.398 \pm 0.001$ & $0.877 \pm 0.004$ & & \\
\hline & 6524.49914 & 0.79535 & $0.57 \pm 0.02$ & $0.399 \pm 0.001$ & $0.879 \pm 0.004$ & $7.4 \pm 0.9$ & $-1.4 \pm 0.9$ \\
\hline & 6525.44457 & 0.82300 & $0.57 \pm 0.02$ & $0.398 \pm 0.001$ & $0.878 \pm 0.004$ & $6.7 \pm 1.0$ & $-1.1 \pm 1.0$ \\
\hline \multirow{14}{*}{2014.61} & 6853.55493 & 0.41687 & $0.56 \pm 0.04$ & $0.399 \pm 0.001$ & $0.892 \pm 0.005$ & $6.8 \pm 1.5$ & $-1.6 \pm 1.5$ \\
\hline & 6856.55743 & 0.50466 & $0.55 \pm 0.03$ & $0.398 \pm 0.001$ & $0.894 \pm 0.004$ & $5.9 \pm 1.1$ & $-1.4 \pm 1.1$ \\
\hline & 6861.57410 & 0.65135 & $0.55 \pm 0.02$ & $0.400 \pm 0.001$ & $0.886 \pm 0.004$ & $8.0 \pm 0.9$ & $0.4 \pm 0.9$ \\
\hline & 6864.55546 & 0.73852 & $0.56 \pm 0.02$ & $0.400 \pm 0.001$ & $0.885 \pm 0.004$ & $5.0 \pm 0.9$ & $-0.7 \pm 0.9$ \\
\hline & 6865.53839 & 0.76726 & $0.56 \pm 0.02$ & $0.401 \pm 0.001$ & $0.885 \pm 0.003$ & $5.3 \pm 0.9$ & $-1.2 \pm 0.9$ \\
\hline & 6866.58350 & 0.79782 & $0.56 \pm 0.01$ & $0.400 \pm 0.001$ & $0.887 \pm 0.003$ & $3.6 \pm 0.9$ & $1.1 \pm 0.9$ \\
\hline & 6881.38890 & 0.23073 & $0.58 \pm 0.02$ & $0.399 \pm 0.001$ & $0.889 \pm 0.004$ & $4.6 \pm 1.1$ & $1.7 \pm 1.1$ \\
\hline & 6889.36216 & 0.46386 & $0.55 \pm 0.03$ & $0.401 \pm 0.002$ & $0.890 \pm 0.006$ & $4.1 \pm 1.7$ & $0.0 \pm 1.7$ \\
\hline & 6893.46397 & 0.58380 & $0.54 \pm 0.02$ & $0.400 \pm 0.001$ & $0.882 \pm 0.003$ & & \\
\hline & 6895.46092 & 0.64219 & $0.54 \pm 0.02$ & $0.399 \pm 0.001$ & $0.887 \pm 0.004$ & $7.5 \pm 1.1$ & $0.9 \pm 1.1$ \\
\hline & 6897.47963 & 0.70121 & $0.54 \pm 0.02$ & $0.398 \pm 0.001$ & $0.878 \pm 0.003$ & $8.6 \pm 0.9$ & $-0.3 \pm 0.9$ \\
\hline & 6899.49512 & 0.76015 & $0.54 \pm 0.01$ & $0.400 \pm 0.001$ & $0.883 \pm 0.003$ & $7.3 \pm 0.9$ & $0.7 \pm 0.9$ \\
\hline & 6900.52575 & 0.79028 & $0.54 \pm 0.01$ & $0.399 \pm 0.001$ & $0.884 \pm 0.004$ & $8.4 \pm 1.1$ & $-0.9 \pm 1.1$ \\
\hline & 6901.48870 & 0.81844 & $0.54 \pm 0.01$ & $0.400 \pm 0.001$ & $0.883 \pm 0.003$ & $7.7 \pm 0.9$ & $-1.4 \pm 0.9$ \\
\hline \multirow{16}{*}{2015.54} & 7200.57599 & 0.56368 & $0.60 \pm 0.03$ & $0.400 \pm 0.001$ & $0.893 \pm 0.004$ & $-0.8 \pm 1.2$ & $0.3 \pm 1.2$ \\
\hline & 7202.53874 & 0.62107 & $0.60 \pm 0.02$ & $0.401 \pm 0.001$ & $0.889 \pm 0.003$ & $-0.5 \pm 0.9$ & $-1.1 \pm 0.9$ \\
\hline & 7203.59939 & 0.65208 & $0.59 \pm 0.03$ & $0.400 \pm 0.001$ & $0.886 \pm 0.004$ & $2.4 \pm 1.0$ & $1.0 \pm 1.0$ \\
\hline & 7204.56304 & 0.68026 & $0.59 \pm 0.03$ & $0.401 \pm 0.001$ & $0.890 \pm 0.004$ & $2.3 \pm 1.0$ & $0.4 \pm 1.0$ \\
\hline & 7211.52277 & 0.88376 & $0.64 \pm 0.03$ & $0.400 \pm 0.001$ & $0.896 \pm 0.004$ & $13.3 \pm 1.0$ & $-1.2 \pm 1.0$ \\
\hline & & & & & $0.890 \pm 0.005$ & $14.1 \pm 0.9$ & $-0.5 \pm 0.9$ \\
\hline & 7213.50574 & 0.94174 & $0.67 \pm 0.02$ & $0.401 \pm 0.001$ & $0.897 \pm 0.003$ & $14.6 \pm 0.9$ & $0.3 \pm 0.9$ \\
\hline & 7214.52800 & 0.97163 & $0.68 \pm 0.05$ & $0.401 \pm 0.001$ & $0.903 \pm 0.005$ & $13.7 \pm 1.0$ & $1.0 \pm 1.0$ \\
\hline & 7215.56071 & 0.00183 & $0.64 \pm 0.03$ & $0.402 \pm 0.001$ & $0.898 \pm 0.004$ & $13.9 \pm 1.0$ & $-0.7 \pm 1.0$ \\
\hline & 7216.54418 & 0.03059 & $0.67 \pm 0.03$ & $0.402 \pm 0.001$ & $0.900 \pm 0.004$ & $14.3 \pm 0.9$ & $-0.9 \pm 0.9$ \\
\hline & 7218.55681 & 0.08943 & $0.69 \pm 0.03$ & $0.403 \pm 0.001$ & $0.906 \pm 0.004$ & $12.4 \pm 1.0$ & $0.5 \pm 1.0$ \\
\hline & 7219.56343 & 0.11887 & $0.69 \pm 0.05$ & $0.403 \pm 0.001$ & $0.906 \pm 0.005$ & $12.0 \pm 1.3$ & $0.2 \pm 1.3$ \\
\hline & 7224.56050 & 0.26498 & $0.68 \pm 0.03$ & $0.401 \pm 0.001$ & $0.904 \pm 0.005$ & $3.5 \pm 1.3$ & $-0.2 \pm 1.2$ \\
\hline & 7240.48208 & 0.73053 & $0.62 \pm 0.04$ & $0.400 \pm 0.001$ & $0.897 \pm 0.005$ & $0.7 \pm 1.3$ & $1.1 \pm 1.3$ \\
\hline & 7245.54843 & 0.87866 & $0.65 \pm 0.04$ & $0.400 \pm 0.001$ & $0.896 \pm 0.005$ & $9.9 \pm 1.4$ & $0.2 \pm 1.4$ \\
\hline & 7246.57034 & 0.90855 & $0.63 \pm 0.01$ & $0.399 \pm 0.001$ & $0.892 \pm 0.004$ & $12.6 \pm 1.0$ & $0.3 \pm 1.0$ \\
\hline
\end{tabular}

